\title{
Sales Growth of New Pharmaceuticals Across the Globe: The Role of Regulatory Regimes
}

\author{
Stefan Stremersch and Aurélie Lemmens
}

\begin{tabular}{|l|l|}
\hline \multicolumn{2}{|l|}{ ERIM REPORT SERIES RESEARCH IN MANAGEMENT } \\
\hline ERIM Report Series reference number & ERS-2008-026-MKT \\
\hline Publication & Mei 2008 \\
\hline Number of pages & 46 \\
\hline Persistent paper URL & http://hdl.handle.net/1765/12340 \\
\hline Email address corresponding author & stremersch@few.eur.nl \\
\hline Address & Erasmus Research Institute of Management (ERIM) \\
& RSM Erasmus University / Erasmus School of Economics \\
& Erasmus Universiteit Rotterdam \\
& P.O.Box 1738 \\
& 3000 DR Rotterdam, The Netherlands \\
& Phone: + 31 10 408 1182 \\
& Fax: + 3110 408 9640 \\
& Email: info@erim.eur.nl \\
& Internet: www.erim.eur.nl \\
\hline
\end{tabular}

Bibliographic data and classifications of all the ERIM reports are also available on the ERIM website: www.erim.eur.nl 


\section{ERASMUS RESEARCH INSTITUTE OF MANAGEMENT}

\section{REPORT SERIES \\ RESEARCH IN MANAGEMENT}

\begin{tabular}{|l|l|}
\hline ABSTRACT AND KEYWORDS \\
\hline Abstract & $\begin{array}{l}\text { Prior marketing literature has overlooked the role of regulatory regimes in explaining } \\
\text { international sales growth of new products. This paper addresses this gap in the context of new } \\
\text { pharmaceuticals (15 new molecules in 34 countries) and sheds light on the effect regulatory } \\
\text { regimes have on new drug sales across the globe. Based on a time-varying coefficient model, } \\
\text { we find that differences in regulation substantially contribute to cross-country variation in sales. } \\
\text { One of the regulatory constraints investigated, i.e. manufacturer price controls, has a positive } \\
\text { effect on drug sales. The other forms of regulation such as restrictions of physician prescription } \\
\text { budgets and the prohibition of direct-to-Consumer advertising tend to hurt sales. The effect of } \\
\text { manufacturer price controls is similar for newly launched and mature drugs. In contrast, } \\
\text { regulations on physician prescription budget and direct-to-consumer advertising have a } \\
\text { differential effect for newly launched and mature drugs. While the former hurts mature drugs } \\
\text { more, the latter has a larger effect on newly launched drugs. In addition to these regulatory } \\
\text { effects, we find that national culture, economic wealth, introduction timing, lagged sales and } \\
\text { competition, also affect drug sales. Our findings may be used as input by managers for } \\
\text { international launch and sales decisions. They may also be used by public policy administrators } \\
\text { to compare drug sales in their country to other countries and to assess the role of regulatory } \\
\text { regimes therein. }\end{array}$ \\
\hline international new product growth, drug, pharmaceutical, regulation, culture, economics, \\
timevarying effects, penalized splines
\end{tabular}




\title{
Sales Growth of New Pharmaceuticals Across the Globe: The Role of Regulatory Regimes
}

Stefan Stremersch and Aurélie Lemmens

Marketing Science

Forthcoming

\begin{abstract}
Aknowledgements
Stefan Stremersch is Chaired Professor of Marketing and the Desiderius Erasmus Distinguished Chair of Economics, Erasmus School of Economics, Erasmus University Rotterdam, and Visiting Professor of Marketing, Fuqua School of Business, Duke University. Aurélie Lemmens is Assistant Professor of Marketing, Erasmus School of Economics, Erasmus University Rotterdam. The authors thank IMS Health for making the data available. They are also grateful to Isabel Verniers, who was willing to share part of the data this study is based on. They acknowledge the support of the Institute of Life Science Business Economics, Erasmus University Rotterdam. Christophe Croux, Bas Donkers, Dennis Fok, Jacob Goldenberg, David Soberman, Walter Van Dyck and participants to the $29^{\text {th }}$ Informs Marketing Science Conference contributed many valuable insights to this research. The authors contributed equally to this article.
\end{abstract}




\title{
Sales Growth of New Pharmaceuticals Across the Globe: The Role of Regulatory Regimes
}

\begin{abstract}
Prior marketing literature has overlooked the role of regulatory regimes in explaining international sales growth of new products. This paper addresses this gap in the context of new pharmaceuticals (15 new molecules in 34 countries) and sheds light on the effect regulatory regimes have on new drug sales across the globe.

Based on a time-varying coefficient model, we find that differences in regulation substantially contribute to cross-country variation in sales. One of the regulatory constraints investigated, i.e. manufacturer price controls, has a positive effect on drug sales. The other forms of regulation such as restrictions of physician prescription budgets and the prohibition of direct-to-consumer advertising tend to hurt sales. The effect of manufacturer price controls is similar for newly launched and mature drugs. In contrast, regulations on physician prescription budget and direct-to-consumer advertising have a differential effect for newly launched and mature drugs. While the former hurts mature drugs more, the latter has a larger effect on newly launched drugs. In addition to these regulatory effects, we find that national culture, economic wealth, introduction timing, lagged sales and competition, also affect drug sales.
\end{abstract}

Our findings may be used as input by managers for international launch and sales decisions. They may also be used by public policy administrators to compare drug sales in their country to other countries and to assess the role of regulatory regimes therein.

Keywords: International new product growth, drug, pharmaceutical, regulation, culture, economics, timevarying effects, penalized splines. 


\section{Introduction}

Over the last decades, marketing research on international new product growth has identified several key drivers of variation across countries. Among them, the most prominently studied are economic and socio-cultural characteristics of nations (Dekimpe et al. 1998, Gatignon et al. 1989, Helsen et al. 1993, Stremersch and Tellis 2004, Talukdar et al. 2002, Tellis et al. 2003, Van den Bulte and Stremersch 2004). Cross-country interrelations have also received special attention, either under the umbrella of cross-national learning, lead-lag or introductory lag effects (see Dekimpe et al. 2000, for an extensive review). However, the role of regulation on new product growth has been mostly overlooked.

This lack of research is particularly surprising as the variety of regulatory systems across the globe was among the prime motivations invoked for international new product growth models (Heeler and Hustad 1980, Mahajan and Muller 1994) and recent calls have been made for more research in this area (e.g. Stremersch and Tellis 2004). Government regulation is an interesting and complex object of study with many societal implications (Shugan 2003). Industries in which regulation affects firms' marketing strategies and sales include telecommunications, energy, tobacco, liquor and financial services. Many countries have regulated the marketing of products in these industries, by means of advertising regulation for tobacco and financial services, price regulation for telecommunications and profit regulations for energy.

In this paper, we focus on regulatory regimes in the pharmaceutical industry as there is increased evidence for its great influence, both from academia (Atun and Gurol-Urganci 2006, Berndt et al. 2005, Chintagunta and Desiraju 2005, Danzon and Ketcham 2003, Kyle 2007; Vakratsas and Kolsarici 2007) and practice (European Commission 2004; Urch 2005). Many different regulatory regimes exist and - while the business press routinely refers to their importance (e.g. The Economist 2007) - their effects on sales are not straightforward and largely 
unexamined. Therefore, pharmaceuticals are an appropriate context to examine the role regulation may play in international sales growth.

Our aim is to model market response to regulation in a non-stationary environment, the new product growth context. Therefore, we consider that regulatory regimes may have a differential effect on drugs that were introduced very recently (e.g. a few months ago) - we refer to such drugs as newly launched drugs - than on maturing drugs that have been on the market for a longer period of time. For instance, regulation that caps the number of prescriptions physicians can write may not influence sales of newly launched drugs that much, while it may seriously depress sales when the drug matures. As we will explain below, the reasons may be that, in the first months after launch, the volume the physician prescribes is still low and is predominantly used by the patients that benefit the most from the new drug, e.g. because alternative treatments for these patients were not effective or showed major side effects. Aside from regulation, prior research on international new product growth also contains some early evidence that other country characteristics, such as national culture and economic wealth affect new product growth differentially across the life cycle (e.g. Stremersch and Tellis 2004, Tellis et al. 2003). Hence, ignoring potential variation over time in the effects of country characteristics, such as regulation, national culture or economic wealth on sales may yield misleading or incomplete insights.

To analyze cross-country variation in new drug sales, we obtained monthly sales data for 15 new molecules, ${ }^{1}$ across 4 therapeutic categories and 34 countries, during their first 84 months (7 years) after launch. To explain the observed international differences, we gather data from many other sources on regulation, culture, and economics. Our data includes many developing nations, in Eastern Europe, Africa, South America, and the Middle East. This provides us with a

\footnotetext{
${ }^{1}$ A molecule is the active substance of a drug. As several competing brands contain the same molecule, we focus on the sales growth pattern of the molecule rather than these brands. This choice is consistent with the literature on new product growth that generally models sales or adoption of a product (e.g. TV), irrespective of the brand (e.g. Sony).
} 
strong empirical basis, though constrained to the context of international sales growth of new pharmaceutical drugs.

The remainder of the paper is organized as follows. In the next section, we discuss the effects of countries' regulatory environments for pharmaceuticals in more detail. In the subsequent sections, we then present our data, the modeling framework employed and our results. Finally, we end with a discussion and suggestions for future research.

\section{The Effect of Regulation on Drug Sales}

It is difficult to parsimoniously conceptualize regulation. In this section, we examine several aspects of the regulatory environment that are salient to marketers. It concerns regulation on: (1) manufacturer price, (2) physician prescription budgets, (3) patient co-payment, (4) marketing efforts to physicians; and (5) direct-to-consumer advertising.

\section{Manufacturer Price Regulation}

While several studies have analyzed the impact of price regulation on launch delays of new drugs (Atun and Gurol-Urganci 2006, Danzon et al. 2005, Kyle 2007), there is - to the best of our knowledge - little evidence as to the effect of price regulation on sales growth of new drugs. Governments may exert price control in multiple ways. The most prominent one is to directly control the manufacturer price for drugs (manufacturer price control). Governments impose these price restrictions regardless of $R \& D$ and production expenses. For instance, many European countries (Belgium, Finland, Greece, Ireland, Luxembourg, the Netherlands, Norway, Portugal and Switzerland) have direct manufacturer price controls. Often, the manufacturer is required to provide clinical evidence of the cost effectiveness of a new drug (e.g. in qualityadjusted-life-years (QALY) added to patients suffering from the disease ${ }^{2}$ ). Based on these cost-

\footnotetext{
${ }^{2}$ QALY is a measure for the beneficial effects of a medical treatment. It is based on the number of life
} years added thanks to the medical treatment. Each year in perfect health is assigned a value of 1, while 
effectiveness studies, the regulated manufacturer price of the new drug is set in a negotiation between the government and the manufacturer. This regulated price will be applied in the marketplace and reviewed periodically, either at the initiative of the regulator or at the manufacturer's initiative (e.g. Urch 2005). Note that the regulator may compensate part of the losses incurred by the manufacturer when a regulated price is lower than the price that would have been set by the manufacturer otherwise.

The aim of direct manufacturer price controls is to lower drug prices. Based on the average 1992 prices of a sample of about 400 drugs across 6 countries, Danzon and Chao (2000) have also shown that average prices of drugs are lower in countries with price controls than in countries without price controls. Wright (2004) has argued theoretically that the regulated price in such direct regulation is also below the price manufacturers would set when there would not be any price controls. Previous research has shown negative elasticities of sales to prices in pharmaceutical markets (Berndt et al. 2002, Danzon and Chao 2000), as physicians often take the cost of the treatment into account in their joint decision with the patient (e.g., Hart et al. 1997). Therefore, we expect price regulation to positively affect sales.

However, such effect may be limited for newly launched molecules, as compared to maturing molecules. Inspiration for such expectation comes from prior literature that examined the link between price regulation and pricing patterns over the new drug's life cycle. This literature has shown that prices fall faster when prices are regulated, than when prices are not regulated (Danzon and Chao 2000; Ekelund and Persson 2003).

Interestingly, we also obtained anecdotal evidence for the pricing pattern we hypothesize above for a subset of the countries in our sample. ${ }^{3}$ Figure 1 shows the typical pricing pattern,

death is assigned a value of 0 . Each year in imperfect health (e.g. because of the loss of a limb, lack of mobility, etc.) is assigned a value between 0 and 1 .

${ }^{3}$ We obtained anecdotal evidence on pricing and marketing efforts for all drugs in our sample, but for a limited set of countries - Belgium, Canada, Germany, Switzerland, the United Kingdom and the United States - for which our data supplier had complete information. 
together with 95\% confidence bounds (see online appendix for computational details) of new drugs in three countries with price control (Belgium, Switzerland, and Canada) and three countries without price control (Germany, the United Kingdom and the United States), over the data window. The values on the $\mathrm{Y}$-axis represent the average drug price pattern across all drugs in these five countries in the presence or absence of regulation, expressed in dollars per gram.

Figure 1 shows that prices in countries with price regulation decrease faster than prices in countries without price regulation, reaching substantially lower levels for maturing drugs. The upward slope in the non-regulated countries is likely due to the inflation.

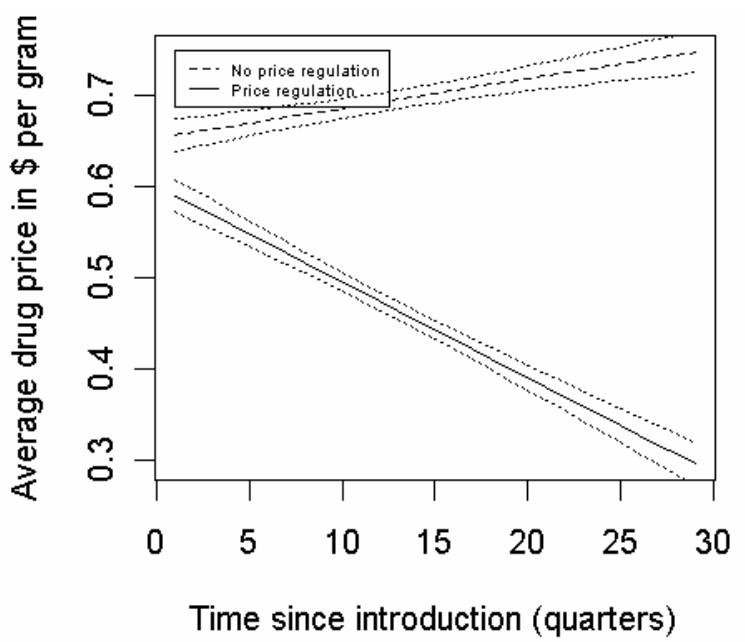

Figure 1: The typical price pattern for new drugs in countries that do not regulate prices versus countries that regulate prices.

For all the reasons posited above, we may expect that, while manufacturer price regulation may increase sales of maturing drugs, it would in turn have little or no effect on sales of recently introduced drugs.

\section{Regulation on Physician Prescription Budgets}

The most direct way in which regulators can intervene in pharmaceutical markets for prescription drugs is to limit the total number of prescriptions a physician can write. For instance, Germany in the early 90s introduced a collective budget for prescription drugs that was later reformed to physician-level prescription budgets. Physicians in Latvia are assigned a fixed budget 
calculated on the number of patients in their practice. Assigning budgets to physicians for the total number of drugs prescribed restricts prescription drug sales. Thus, countries that impose such restrictions are likely to show lower drug sales than countries that do not.

Typically, physicians adopt a stepwise approach in prescription (Prosser et al. 2003). They try familiar alternatives first, and only prescribe new drugs if the familiar alternatives fail or cause adverse effects. Prosser and Walley (2003) find that physicians typically prescribe newly introduced drugs to patients who do not undergo significant improvement in their (mostly severe) condition when using prior alternatives or who do not tolerate such alternatives because of side effects that occur. Therefore, the effect of prescription budget regulation on early sales of a newly launched drug may be limited. However, the total market potential for the drug may be smaller in the presence of prescription budget regulation. In regulated markets, physicians will feel more pressure not to prescribe the drug to patients with mostly mild symptoms or who tolerate traditional alternatives well, as compared to non-regulated markets.

\section{Patient Co-Payment Regulation}

In many countries, patients are required some form of co-payment for prescription drugs. For instance, Belgian patients typically pay only a small part of their prescription medicine (the rest is paid by a government institution) and the contribution they pay is set at the level of the individual drug (again taking into account QALY). Hungary has a similar system (although the co-payment amounts differ), but also adds patient income to the reimbursement level, with higher reimbursements (in many cases $100 \%$ ) for the poor. In other countries, patients do not co-pay for drugs. For instance, in the Netherlands, the cost of prescription drugs and physician visits is directly settled between physician, pharmacist and the patient's insurance company (which can be private or public). In co-payment systems, patients are monetarily sanctioned for drug use and thus experience a part of the cost of their treatment directly (Reuveni et al. 2002). Such monetary 
sanctioning is expected to depress drug sales, given that it makes treatment cost a more salient criterion in the physician-patient joint decision (e.g. Hart et al. 1997).

A policy initiative in Italy provides initial face validity for the above reasoning. Italy abolished its co-payment system at the end of 2000. In the first few months of 2001, pharmaceutical spending in Italy rose by anything up to $30 \%$ (while the federation of Italian family practitioners assessed the total increase to be around 2-3\%). Soon after, co-payments were reintroduced by the Italian government (Urch 2005). Cross-country variation in drug sales, driven by the absence or presence of a co-payment system, has not been investigated upon such a large scale as in the present paper.

There is no reason to expect the presence of a co-payment system to affect newly launched drugs differently than mature drugs, as the level of co-pay in countries may not depend on the drug's age or, even, on the drug's price. For example, in Austria, patients pay a flat fee of $€ 4.45$ per prescription. In Portugal, patients pay a fixed percentage of the drug cost according to the essential nature of the drug (specified at the category level).

\section{Regulation on Marketing Efforts to Physicians}

Marketing activities to physicians represent a large and growing part of the expenditures in the pharmaceutical industry. Mizik and Jacobson (2004) indicate that U.S. companies have spent over $\$ 5.8$ billion in 2002 on detailing, combined with another $\$ 11.5$ billion on free sampling. In view of these large costs, scholars have paid a lot of attention to the effects of marketing efforts to physicians on prescription behavior and pharmaceutical sales (Chintagunta and Desiraju 2005, Dekimpe and Hanssens 1999, Ding and Eliashberg 2008, Hahn et al. 1994, Leeflang et al. 2005, Manchanda and Chintagunta 2004, Manchanda and Honka 2005, Manchanda et al. 2004, Mantrala et al. 1994, Narayanan 2004, Narayanan et al. 2004, Narayanan et al. 2005). There has been some debate on the direction of the effect of marketing efforts on prescription behavior. Some authors find positive effects (e.g. Gönül et al. 2001), others find 
neutral effects (e.g. Rosenthal et al. 2003), and still others find negative effects (e.g. Parsons and Vanden Abeele 1981). Also the size of these effects has been under debate; some find strong effects (e.g. Gönül et al. 2001), while others find more modest effects (e.g. Mizik and Jacobson 2004). Nonetheless, the overall conclusion is that marketing efforts often have a positive and significant effect on physician decision making. Patients are typically not aware of this influence on the prescription decision.

The marketing efforts that prior literature has considered are: detailing, meetings, and sampling (see, Narayanan and Manchanda 2006, Venkataraman and Stremersch 2007). Regulation may constrain the number of detailing visits a manufacturer can make, the number of meetings a manufacturer can organize, or the number of samples a manufacturer can dispense to physicians. For instance, the amount manufacturers spend in the United Kingdom on sales promotions is limited to a proportion of their overall (not drug-specific) profits (the "marketing allowance"). In Spain, promotional costs are capped at a percentage of sales of the firm. Given the expected positive effect of marketing efforts on new drug sales, we expect that countries that limit the marketing efforts of a pharmaceutical firm to physicians would show lower drug sales than countries that do not impose such limits.

One may counter that if the cap is quite high, it may not affect marketing efforts at all, and therefore not affect drug sales. However, this argument does not fit the regulatory practices of countries. First, the reason why governments implement these caps to marketing efforts is always to limit prescriptions (see Urch 2005). Second, just from the specific regulations, one can appreciate the low level at which marketing efforts are capped. For instance, in Poland, manufacturers can only drop, at maximum, 3 samples of the smallest package with a physician's office over an entire year. Such approach contrasts sharply with the United States, where it is common to drop in the neighborhood of 10 samples with a physician's office per year (source: IMS Health, category for anti-arthritic treatments $(\mathrm{Cox}-2))$. 
Anecdotal evidence we obtained for a subset of the countries confirms the negative influence of such regulation on marketing efforts. Similar to Figure 1, we report the typical pattern of marketing efforts for new drugs, together with $95 \%$ confidence bounds, in two countries with restrictions on marketing efforts (Belgium and the United Kingdom) and three countries without such restrictions (Germany, Switzerland and the United States) in Figure 2. The values on the $\mathrm{Y}$-axis represent the average pattern in marketing efforts across all drugs in these five countries in the presence or absence of regulation, expressed in thousands of dollars. It shows that marketing efforts are lower in countries with restrictions on marketing efforts than in countries without such restrictions.

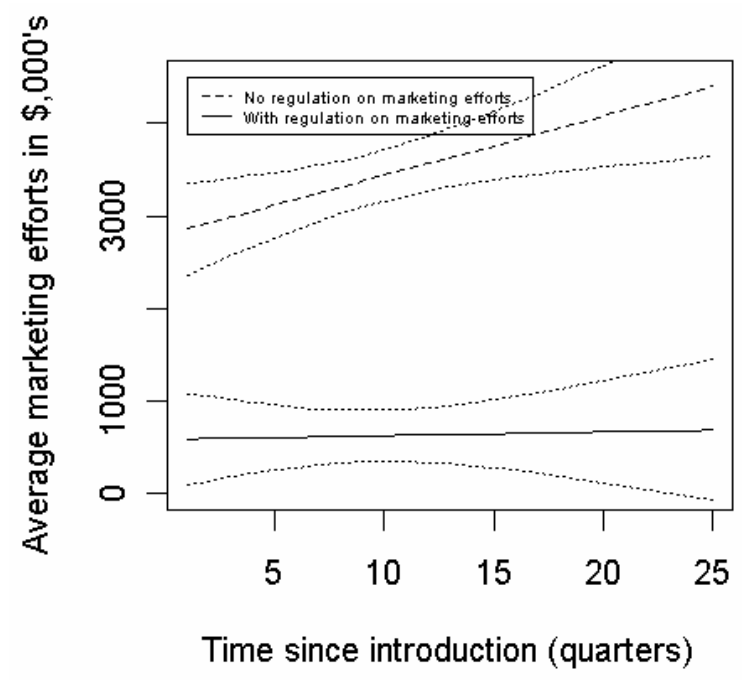

Figure 2: The typical pattern in marketing efforts for new drugs in countries that do not restrict marketing efforts versus countries that restrict marketing efforts.

In addition, one may expect that the restriction of marketing efforts to physicians has a larger effect for newly drugs than for maturing drugs. The literature on advertising has shown that the sales-to-advertising elasticity decreases over a product's life cycle (Chandy et al. 2001; Tellis and Fornell 1988). The reason is that consumers are more motivated to process information about new products than older ones (Grunert 1996). The same very likely applies to physicians, as Neslin (2001) and Narayanan et al. (2005) show that detailing is the most effective in the introduction phase. In consequence, a regulatory restriction is likely to depress sales of newly 
launched drugs more than sales of maturing drugs. A potential counterargument may be that firms could increase their marketing efforts to physicians as the drug matures. However, data on the pattern of communication efforts over time generally do not support this argument; rather, they support the contrary (for examples, see Horsky and Simon 1983; Lilien and Little 1976). In the pharmaceutical industry, Narayanan et al. (2005) show that market efforts to physicians for antihistamines increase for Allegra, decrease for Zyrtec and remain constant for Claritin. Also the anecdotal evidence in Figure 2 shows stable marketing efforts over time.

\section{Regulation on Direct-to-Consumer Advertising (DTCA)}

While regulating marketing efforts to physicians is a direct way to affect physicians' prescription behavior, pharmaceutical firms can also attempt to influence patients by advertising the drug directly to consumers. So far, only two countries, New Zealand and the United States, allow pharmaceutical marketers to directly advertise to consumers. The practice is more novel than marketing efforts to physicians as it only became legal to directly advertise to consumers in the eighties in the United States and in the early nineties in New Zealand. Yet, the instrument is quickly becoming very popular among marketers. Spending on DTCA in the United States, for example, has increased from \$1.1 billion in 1997 to $\$ 5.6$ billion in 2006, according to IMS Health.

Prior literature has shown that DTCA triggers patients - generally 10 to $44 \%$ of the people that viewed the ad - to request drugs by brand name from their physicians (Cohen 1988; Mehta and Purvis 2003; Mintzes et al. 2003; Rosenthal et al. 2003; Weissman et al. 2004; West 1999; Wosinska 2002). Such patient requests are often accommodated by physicians, mainly because it affects physician visit satisfaction (Kravitz et al. 2003) and it shows patients that the physician cares (Schwartz et al. 1989). For instance, Bell et al. (1999) found that $46 \%$ of patients thought they would be disappointed if a physician did not prescribe an advertised drug they 
requested, while $24 \%$ thought they might attempt to obtain the prescription from a different doctor and $15 \%$ thought they would switch to a new doctor.

Given the expected positive effect of DTCA on drug sales, we expect that countries that forbid DTCA would show lower drug sales than countries, such as New Zealand and the United States, that do not impose such a restriction. We expect this effect to become smaller as a drug matures, for reasons similar to those developed on restrictions in marketing efforts to physicians. More precisely, we expect higher DTCA effectiveness for new drugs than for maturing drugs, in line with prior results by Neslin (2001) and consistent with the argument above regarding consumers' higher motivation to process information about new products than old ones. Moreover, DTCA is generally high for newly launched drugs and declines as the drug matures (Rosenthal et al. 2002).

\section{Other Variables}

When examining the role of regulation in the context of new pharmaceuticals, we need to control for other factors that may explain cross-national variation in drug sales. Building our research on existing international new product growth literature, we subsequently consider the role of national culture (Stremersch and Tellis 2004, Tellis et al. 2003, Van den Bulte and Stremersch 2004), economics (Talukdar et al. 2002, Van den Bulte and Stremersch 2004), introduction lag (Dekimpe et al. 2000, Kumar and Krishnan 2002, Talukdar et al. 2002), lagged sales (Desiraju et al. 2004) and competition (Chintagunta and Desiraju 2005, Desiraju et al. 2004) in the sales growth of new pharmaceuticals.

\section{National Culture}

Geert Hofstede $^{4}$ (Hofstede 1980 and 2001) identified four main dimensions of national culture that can be related to new drug sales: (1) uncertainty avoidance; (2) individualism; (3)

\footnotetext{
${ }^{4}$ An alternative operationalization is Schwartz's (1992) model of personal values. Hofstede's national culture framework is the framework that is most often adopted by scientists, also in marketing. Early March
} 
masculinity and (4) long term orientation. ${ }^{5}$ Hofstede's national culture dimensions are widely accepted and frequently used for cross-country comparisons (e.g., Dawar and Parker 1994, Lynn et al. 1993, Roth 1995, Steenkamp et al. 1999).

Uncertainty avoidance refers to the extent to which the population of a country feels (intolerable) anxiety when facing uncertain or unknown situations (Hofstede 2001). Patients in uncertainty avoidant cultures show lower subjective health (Hofstede 2001). Lower subjective health perceptions may lead to higher demand for pharmaceutical drugs, considering that physicians typically accommodate patients' requests for treatment (Bell et al. 1999). However, the positive effect of uncertainty avoidance on drug sales may only materialize towards maturing drugs, and have little or no effect on newly launched drugs. Indeed, new products are usually perceived as more risky, and their performance more ambiguous than established products and brands (Steenkamp et al. 1999). In cultures of high uncertainty avoidance, consumers are more likely to identify uncertainty in new products, compared to consumers in low uncertainty avoidance cultures (Michaut 2004). Moreover, societies high in uncertainty avoidance consider novel ideas as dangerous and are more intolerant toward change than societies low in uncertainty avoidance (Hofstede 1980). This may temper the positive effect of uncertainty avoidance on drug sales when it turns to newly launched drugs.

Individualism describes the relationship between the individual and the collectivity that prevails in a given society and to what extent people prefer to act as individuals rather than as members of a social group (Hofstede 2001). Individualism may have several effects on drug sales. In general, individualist cultures tend to spend more of their public and private budgets on

2007, the top three cited references of Hofstede totaled 6,741 citations, while the top three works of Schwartz totaled only 1,035 citations (source: Google Scholar). Obviously, our work can easily be extended to test for the effects of Schwartz's model of personal values, using the same methodology. ${ }^{5} \mathrm{We}$ found neither theoretical support nor empirical evidence in our data to include power distance, the fifth Hofstede dimension, as explanatory variable. While power distance may very well be related to the structure of the health system (for instance, one may imagine medical doctors and pharmacists to take a more hierarchical position towards their patients in power distant cultures), there seems to be no argument to relate it to new drug sales. 
health care (Hofstede 2001), which fits with the emphasis that individualist cultures put on individual well-being. In contrast, individual well-being in collectivist cultures is only relevant in relation to the collective well-being. Higher spending on health care will lead to higher drug sales. Patients in individualist societies also seek doctor consultation more actively than patients in collectivist societies, which, in turn also results in higher drug sales. While collectivist cultures have on average lower drug usage, the difference may be less pronounced for newly launched drugs. Indeed, as already stated above, newly launched drugs are typically prescribed to patients with a strong need for new therapeutical treatments, either because they did not undergo significant improvement in their condition when using prior alternatives or did not tolerate them because of side effects. Thus, differences in drug sales between collectivist and individualist cultures may be less apparent for newly launched drugs and only materialize for maturing drugs.

Masculinity refers to the extent to which the value system, shared by the majority of the members of the middle class of a society, is characterized by male or female attributes (Hofstede 1980). In masculine societies, males are supposed to be tough, while women are supposed to be tender. In feminine societies, both men and women are supposed to be tender (Hofstede 2001). Members of masculine societies, including patients and physicians, conceive the need for medical care as a weakness and a cause for shame when adhering to a masculine value system (Weber et al. 2000). In such societies, we can expect males to seek or receive medical treatment less than males in feminine societies.

Long-term orientation opposes long-term to short-term aspects of Confucian thinking: persistence and thrift to personal stability and respect for tradition (Hofstede 2001). Persistence, perseverance and having a sense of shame are important values associated with a long-term orientation. Therefore, societies high in long-term orientation value typical Confucianist virtues, such as persevering, working hard, being patient and refraining from conspicuous consumption (Hofstede 2001). Members of societies high in long-term orientation may therefore refrain from drug therapy to treat certain ailments, such as high lipids or erectile dysfunction, but rather show 
a different response to such ailments. A more acceptable decision for patients and physicians as a reaction to the cardiovascular risks of high lipids in countries high in long-term orientation may be changing food behaviors and exercising, rather than statin therapy. Also erectile dysfunction would be a cause for shame in many societies high in long-term orientation; one would accept not being able to have sex anymore more readily than in societies low in long-term orientation that are more focused on immediate gratification. Thus, sales of new drugs may be lower in countries high in long-term orientation than in countries low in long-term orientation.

\section{Economic Wealth}

Economic wealth has often been advanced as the major economic characteristic of nations that affects new product sales growth. The economic wealth of nations, most commonly operationalized by GDP per capita, may have several positive effects on sales of pharmaceuticals. On the one hand, the population of more wealthy countries can more easily afford drugs, than the population of less wealthy countries (Desiraju et al. 2004, Stremersch and Tellis 2004, Talukdar et al. 2002, Tellis et al. 2003, Van den Bulte and Stremersch 2004). On the other hand, economic wealth is also associated with better health infrastructure, which in turn may positively affect the sales of new drugs (Desiraju et al. 2004). In our view, these arguments are likely to play similarly for newly launched and maturing drugs.

\section{Cross-Country Introductory Lag}

Cross-country introduction delays refer to the time elapsed between the first introduction of a new product in any country of the world and its introduction in a focal country (Talukdar et al. 2002). The uptake of new molecules may be faster in laggard countries than in lead countries, because patients and physicians learn from experiences in lead countries (Dekimpe et al. 2000, Desiraju et al. 2004, Takada and Jain 1991). These cross-country "learning effects" have been found to generalize to multiple types of decision makers, including consumers, firms and 
governments (Dekimpe et al. 2000). However, previous work also suggests that firms are likely to delay entry in countries that they judge "less attractive" than others. Factors influencing such a delay may be regulation (e.g. Kyle 2007) or expected sales. As such, introduction lag is not exogenous to our model. As further explained in the Model section, we solve this issue by using an instrumental variable procedure.

Cross-country learning effects are likely to vary over time. The existence of crosscountry learning effects can be explained by the role they play in reducing the uncertainty associated with the adoption of new products. As new products are generally associated with more uncertainty than established products and brands (Steenkamp et al. 1999), we therefore expect that the role of cross-country learning effects will be more pronounced for newly launched than for maturing drugs.

\section{Competition}

The presence of competitive firms and brands has often been neglected in the previous new product growth literature, but is now largely recognized to be of major influence on new product sales (Chintagunta and Desiraju 2005, Mahajan et al. 1993, Mason 1990, see Chatterjee et al. 2000, for a review). To account for the role of word-of-mouth communication and the substitution dynamics between competing brands, Mahajan et al. (1993) proposed a diffusion modeling approach assessing the impact of competitive entry on market size and the sales of incumbent firms. Recently, Krishnan et al. (2000) found that the entry of a competing brand in the cellular telephone industry can affect the total market potential of the category and/or the speed of diffusion in the category, depending on the market studied. In the pharmaceutical industry, early evidence for the antidepressant category (Desiraju et al. 2004) suggests that competition may not have a strong influence. Given these mixed findings, it is hard to make expectations as to the direction of this effect, as well as to its variation over time. 


\section{Lagged Sales}

There are three reasons why lagged sales of a new drug may affect present sales of that drug. First, once a physician starts prescribing a new drug, he may trigger adoption also by other physicians, an effect commonly known as contagion. Second, physicians show considerable inertia in prescription behavior (Janakiraman et al. 2005; Venkataraman and Stremersch 2007). Thus, physicians that heavily prescribe a certain drug today are likely to maintain a similar prescription behavior in the future. Third, in the case of chronic diseases, patients generally stay on the same drug for a longer time, receiving refill prescriptions repeatedly. All three effects will create duration dependence in sales, either coming from influence across physicians, persistence over time within a physician's prescription behavior, or persistence over time within a patient's treatment behavior.

Contagion among physicians and inertia in the physicians' prescription behaviors are typically phenomena that affect already established drugs, rather than newly launched drugs. Thus, we expect the effect of lagged sales to increase over the new drugs' life cycle.

We provide an overview of all our expectations in Table 1. In columns 2 and 3, we define the operationalization of the variables. In columns 4 and 5, we specify the effects expected for each covariate studied. In particular, column 4 specifies the main effect, while column 5 specifies how the effect is likely to change over time as the drug matures. We also include our empirical findings in columns 6 and 7 and assess whether our expectations are confirmed in the last column. 
Table 1: Summary table of variable operationalization, expectations and empirical findings

\begin{tabular}{|c|c|c|c|c|c|c|c|}
\hline & \multicolumn{2}{|c|}{ Operationalization of the variable } & \multicolumn{2}{|c|}{$\begin{array}{c}\text { Expected effect on sales in kg } \\
\text { per 1,000 inhabitants aged } 15 \\
\text { years and older }\end{array}$} & \multicolumn{2}{|c|}{$\begin{array}{c}\text { Found effect on sales in } \mathrm{kg} \\
\text { per 1,000 inhabitants aged } \\
15 \text { years and older }\end{array}$} & \multirow[t]{2}{*}{$\begin{array}{c}\text { Were } \\
\text { expectation } \\
\text { confirmed? }\end{array}$} \\
\hline & Variable definition & $\begin{array}{c}\text { Time-varying or } \\
\text { time-invariant } \\
\text { variable }\end{array}$ & $\begin{array}{l}\text { Main } \\
\text { effect }\end{array}$ & $\begin{array}{c}\text { Expected direction } \\
\text { of change over time }\end{array}$ & $\begin{array}{l}\text { Main } \\
\text { effect }\end{array}$ & $\begin{array}{c}\text { Time } \\
\text { variation }\end{array}$ & \\
\hline \multicolumn{8}{|l|}{ Regulation } \\
\hline Manufacturer Price & $\begin{array}{l}\text { Existence of a direct control of the ex- } \\
\text { manufacturer price }\end{array}$ & Time-varying & + & + & + & N.s. & Partly \\
\hline $\begin{array}{l}\text { Physician Prescription } \\
\text { Budgets }\end{array}$ & $\begin{array}{l}\text { Existence of a restriction on the total number } \\
\text { of prescriptions a physician can write }\end{array}$ & Time-varying & - & - & - & - & Yes \\
\hline Patient Co-Payment & $\begin{array}{l}\text { Participation of the patient in the payment of } \\
\text { prescription drugs }\end{array}$ & Time-varying & - & N.s. & N.s. & N.s. & Partly \\
\hline $\begin{array}{l}\text { Marketing Efforts to } \\
\text { Physicians }\end{array}$ & $\begin{array}{l}\text { Existence of a restriction on the number of } \\
\text { detailing visits, meetings and samples }\end{array}$ & Time-varying & - & + & N.s. & N.s. & No \\
\hline $\begin{array}{l}\text { Direct-to-Consumer } \\
\text { Advertising }\end{array}$ & $\begin{array}{l}\text { Prohibition of direct-to-consumer advertising } \\
\text { by the government }\end{array}$ & Time-varying & - & + & - & + & Yes \\
\hline \multicolumn{8}{|l|}{ Other variables } \\
\hline Uncertainty Avoidance & $\begin{array}{l}\text { Extent to which members of a society attempt } \\
\text { to cope with uncertainty and ambiguity }\end{array}$ & Time-invariant & + & + & N.s. & + & Partly \\
\hline Individualism & $\begin{array}{l}\text { Degree to which individuals are expected to } \\
\text { stand up for themselves and are integrated } \\
\text { into groups }\end{array}$ & Time-invariant & + & + & + & + & Yes \\
\hline Masculinity & $\begin{array}{l}\text { Extent to which the value system, shared by } \\
\text { the majority of the members of the middle } \\
\text { class of a society, is characterized by male or } \\
\text { female attributes }\end{array}$ & Time-invariant & - & N.s. & - & N.s. & Yes \\
\hline Long-Term Orientation & $\begin{array}{l}\text { Describes a society's time horizon, or the } \\
\text { importance attached to the future versus the } \\
\text { past and present }\end{array}$ & Time-invariant & - & N.s. & - & N.s. & Yes \\
\hline Economic Wealth & GDP per capita & Time-varying & + & N.s. & + & N.s. & Yes \\
\hline $\begin{array}{l}\text { Cross-Country Introductory } \\
\text { Lag }\end{array}$ & $\begin{array}{l}\text { Number of months elapsed since the first } \\
\text { introduction of the drug in any country }\end{array}$ & Time-invariant & $+/-$ & - & N.s. & - & Partly \\
\hline Competition & $\begin{array}{l}\text { Number of competitive molecules in the same } \\
\text { ATC category of the drug in the country }\end{array}$ & Time-varying & $+/-$ & $+/-$ & - & - & Partly \\
\hline Lagged Sales & $\begin{array}{l}\text { Sales of the drug in the country one period } \\
\text { back }\end{array}$ & Time-varying & + & + & + & + & Yes \\
\hline
\end{tabular}




\section{Data}

We studied the sales growth process of 15 new molecules in four categories that were launched over the period 02/1994 to 12/2004. The four categories (ATC Codes ${ }^{6}$ in-between brackets) are: lipid modifying agents, more specifically statins (C10aa), urinary antispasmodics (G4bd), erectile dysfunction drugs (G4be), and (other) antihistamines for systematic use (R6ax). We selected these categories for several reasons. First, these drug categories represent a substantial portion of the total pharmaceutical market in dollar sales (around 10\%). Second, they are typically prescribed by general practitioners, rather than administered in a hospital (e.g. oncology). In the drug categories we study, retail sales represent more than $98 \%$ of the total volume, the remaining sales being made through the 'in-patient' hospital channel and direct government purchases. Third, these drug categories contain a sufficient number of new drugs that entered after the start of the observation window, which is fixed to $02 / 1994$ because of data handling procedures of IMS Health. We consider the sales growth process across 34 countries that span the globe (see Figure 3 below), including both developed and developing countries.

The country-level sales data consists of quantity sold in grams in retail, per 1,000 inhabitants aged 15 years and above. Such normalization corrects for country size, as advocated by Dekimpe et al. (1998), and also accounts for the fact that the drugs considered are mainly designed for the adult population. This correction is particularly relevant as we consider together developed and developing countries for which the age distribution may strongly differ. ${ }^{7}$ Fifteen years and above is a typical cut-off in international statistics, such as the UN or OECD, and is used by other scholars in the context of international pharmaceutical markets (Lanjouw 2005). We obtained the sales data from IMS Health for each molecule from its market introduction, with the maximum number of months for drug-country combinations being 84 months. Census data

\footnotetext{
${ }^{6}$ More information on the Anatomical Therapeutic Chemical (ATC) codes can be found on the official website of the WHO Collaborating Centre for Drug Statistics Methodology: http://www.whocc.no/atcddd/. ${ }^{7}$ We thank one of the reviewers for this insight.
} 
are gathered from the United Nations Statistical Division website. Table 2 provides an overview of therapeutic categories, their molecules with commercial brand names (one molecule can be sold under different brand names and by different companies), and the period in which the molecule was launched for the first time.

The healthcare regulatory data includes annual data from multiple sources. The first source is URCH Publishing. URCH Publishing is an independent information provider for the biotechnological, chemical, and pharmaceutical industries. The second source is the OECD, which publishes reports on health regulation among its member states (e.g. Jacobzone 2000). The third source includes local health officials and ministries of health, contacted individually.

We operationalize regulation through five dummy variables. The first dummy variable (manufacturer price regulation) takes the value 1 if the government directly controls the manufacturer price for drugs, 0 otherwise. The second dummy variable (regulation on marketing efforts to physicians) takes the value 1 if the government has regulated the number of detailing visits a manufacturer can make, the number of meetings a manufacturer can organize, or the number of samples a manufacturer can dispense to physicians, 0 otherwise.

\begin{tabular}{cccc}
\hline $\begin{array}{c}\text { ATC } \\
\text { code }\end{array}$ & Molecule & Brands & $\begin{array}{c}\text { Time of first } \\
\text { introduction }\end{array}$ \\
\hline C10aa & Atorvastatin & Lipitor & $1 / 1 / 1997$ \\
& Cerivastatin $^{8}$ & Baycol, Lipobay & $4 / 1 / 1997$ \\
& Fluvastatin & Lescol & $2 / 1 / 1994$ \\
& Rosuvastatin & Crestor & $2 / 1 / 2003$ \\
G4bd & Solifenacin & Visicare & $9 / 1 / 2004$ \\
& Tolterodine & Detrol & $11 / 1 / 1997$ \\
G4be & Alprostadil & Caverject, Muse, Viridal & $3 / 1 / 1994$ \\
& Apomorphine & Uprima, Ixense & $6 / 1 / 2001$ \\
& Sildenafil & Viagra, Revatio, Caverta & $1 / 1 / 1998$ \\
& Tadalafil & Cialis & $1 / 1 / 2003$ \\
& Vardenafil & Levitra & $3 / 1 / 2003$ \\
R6ax & Desloratadine & Clarinex & $1 / 1 / 2001$ \\
& Epinastine & Elestat & $6 / 1 / 1994$ \\
& Fexofenadine & Allegra, Telfast & $8 / 1 / 1996$ \\
& Mizolastine & Mizollen & $1 / 1 / 1998$ \\
\hline
\end{tabular}

Table 2: Our sample of pharmaceutical drugs

\footnotetext{
${ }^{8}$ Note that Cerivastatin was withdrawn from the market in 2001 . We only include sales data of this molecule until the withdrawal announcement to avoid a structural break in the sales data.
} 
The third dummy variable (regulation on physician prescription budgets) takes the value 1 if regulation restricts the prescriptions a physician can write, 0 otherwise. The fourth dummy variable (patient co-payment regulation) takes the value 1 if patients are required some form of co-payment for prescription drugs, 0 otherwise. The fifth dummy variable (regulation on directto-consumer advertising) takes the value 1 if direct-to-consumer advertising is prohibited, 0 when it is allowed.

While the regulatory environment is intrinsically more complex than the presence or absence of a restriction, it is hard to obtain more detailed information on the national peculiarities for each of the regulatory variables, and to code them in a sound and economical manner.

Therefore, other scholars have also resorted to a dummy coding, in a manner similar to ours (Kyle 2007). Yet the combination of these five regulatory dimensions still offers us $2^{5}$, or 32 possible regulatory frameworks, of which 13 actually occur in practice. We also find quite a significant cross-national diversity in terms of each of the regulatory variables. For instance, about $65 \%$ of the countries have manufacturer price regulation; $16 \%$ regulate the marketing efforts to physicians; 37\% limit physicians' prescription budgets; 75\% apply a patient co-payment system; and $96 \%$ prohibit direct-to-consumer advertising.

As readers may be relatively unfamiliar with countries' regulation profiles, Figure 3 summarizes all regulation dummies in a composite regulation score (from "no regulation" (on all of the above five aspects), to "fully regulated" (on all of the above five aspects)), on which we position each of the countries. The interior points are determined by the number of regulatory restrictions in effect. This ranking has no other ambition than illustrating the intensity of the national regulatory regimes to the reader and, for simplicity, makes no qualitative distinction between the various regulatory aspects. ${ }^{9}$ The position of countries may change, if they made

\footnotetext{
${ }^{9}$ While our model in the results section includes dummies for each of the five different regulations, we are not able to disclose them at the individual country level because this data is, in part, proprietary. For more detail on each country's regulatory environment, we refer readers to Urch, which publishes many
} 
regulatory changes in the data window. A hyphen following the year means "as of that year", while a hyphen preceding the year means "before that year". Note however that changes in regulation are quite rare in our data window. Only $17 \%$ of the countries included made a regulatory change during the time frame.

For cultural characteristics of countries, we use Hofstede's (2001) values on four cultural dimensions, uncertainty avoidance, individualism, masculinity and long-term orientation. Hofstede's values have been shown to be very robust indicators of national culture (Steenkamp et al. 1999, Tellis et al. 2003). Given the relatively high number of missing values in the long-term orientation dimension, we proceed to the imputation of missing values using the multiple imputation method for continuous data proposed by Schafer $(1997,2002)$. This method has been recently advocated and used in a similar context by Deleersnyder et al. (2008).

\section{No regulation}

United Brazil States

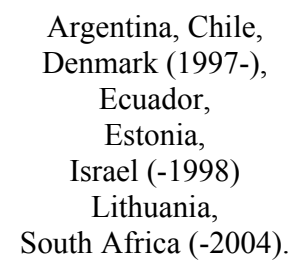

Austria (1999-),
Australia, Czech Republic,
Denmark (-1997),
Finland, Germany,
Israel (1998-),
Italy (-1996),
Latvia, Luxembourg,
Mexico (1996-),
The Netherlands (-1996),
Norway, Poland (-2002),
Portugal, Slovakia,
South Africa (2004-),
Sweden, Turkey.

Fully regulated

Austria (-1999)

Greece,

Hungary, Ireland, Italy (1996-), France (-1996), Mexico (-1996), The Netherlands (1996-), Poland (2002-), Spain, Switzerland, United Kingdom.

\section{Figure 3: Countries from no regulation to fully regulated}

Quarterly GDP per capita is used as a measure of economic wealth, obtained by dividing production-based GDP data from Thomson DataStream at current prices, converted to U.S. dollars, without seasonal adjustment, by United Nations mid-year population data for all 34 countries involved. The introductory lag effect for drug $i$ in country $j$ at time $t$ is operationalized as the number of months elapsed since the first introduction of drug $i$ in any country. This

regulatory reports on international pharmaceutical markets at a relatively low price, and the OECD, which publishes reports on the pharmaceutical regulation of its member states. The regulatory information we gathered ourselves for countries not covered by Urch or OECD can be obtained from the authors upon request. 
variable is time-invariant. The effect of competition for drug $i$ in country $j$ at time $t$ is operationalized as the number of competitive molecules that belong to the same ATC category and that are present/sold in country $j$ at time $t$. To have a complete picture of the market conditions, we also include the molecules introduced before 1994 and that belong to the same ATC category. This variable is time-varying. Finally, lagged sales for drug $i$ in country $j$ at time $t$ is operationalized as the sales of the same drug $i$ in the same country $j$ in $t-1$.

To conclude, note that the tests we conducted (variance inflation factors, condition indices) did not detect severe multicollinearity.

\section{Model Specification}

Let $y_{i c j t}$ be the monthly grams per thousand inhabitants aged 15 years and above of drug $i$ belonging to the drug category (ATC) $c$ sold in country $j$ at time $t$, with $t=1, \ldots, T_{i c j}$, where $T_{i c j}$ is the number of sample points for drug $i$ from category $c$ in country $j$. All categories that we consider are available in every country. We specify a nested structure in which each drug $i$ is nested in a particular drug category $c$. By doing so, we allow for higher correlation among sales of drugs belonging to the same ATC. For all drugs, we have $y_{i c j t}=0$ up to $t=0$. We model $y_{\text {icjt }}$ using the following time-varying coefficient model:

$$
\begin{aligned}
y_{i c j t}= & \beta_{1 t} \operatorname{PRICE}_{j t}+\beta_{2 t} \text { PROM }_{j t}+\beta_{3 t} \mathrm{BUDG}_{j t}+\beta_{4 t} \mathrm{COPAY}_{j t}+\beta_{5 t} \mathrm{DTCA}_{j t}+ \\
& \beta_{6 t} \overline{\mathrm{UA}}_{j}+\beta_{7 t} \overline{\mathrm{IND}}_{j}+\beta_{8 t} \overline{M A S}_{j}+\beta_{9 t} \overline{L T O}_{j}+ \\
& \beta_{10 t} \overline{G D P}_{j t}+\beta_{11 t} \overline{C O M P}_{i c j t}+\beta_{12 t} \bar{y}_{i c j t-1}+\beta_{13 t} \overline{I L}_{i c j}+\eta_{i c j t} \\
\text { with } \eta_{i c j t}= & \alpha_{c}+\gamma_{i c}+\varepsilon_{i c j t}, \alpha_{c} \sim N\left(\alpha_{0}, \sigma_{\alpha}^{2}\right), \gamma_{i c} \sim N\left(0, \sigma_{\gamma}^{2}\right) \text { and } \varepsilon_{i c j t} \sim N\left(0, \sigma_{t}^{2}\right),
\end{aligned}
$$

where the $\beta_{p t}, p=1, \ldots, 13$, are the smoothly time-varying effects of the covariates under investigation. This model allows us to disentangle the effect of each country characteristic on new drug sales from its market introduction until 84 months after introduction. Upon a preliminary visual 
inspection of the residuals, we allow the error term to be heteroskedastic with $\sigma_{t}^{2}=\sigma^{2}|t| \cdot{ }^{10}$ In the above equation, $P R I C E_{j t}$ indicates whether the manufacturer price is regulated $(=1)$ or not $(=0)$ in country $j$ in month $t, P R O M_{j t}$ specifies whether marketing efforts to physicians are regulated (=1) or not $(=0)$ in country $j$ in month $t, B U D G_{j t}$ denotes whether regulation limits physicians' prescription budgets $(=1)$ or not $(=0)$ in country $j$ in month $t, C O P A Y_{j t}$ indicates whether a copayment system is applied $(=1)$ or not $(=0)$ in country $j$ in month $t$, and $D T C A_{j t}$ stands for the interdiction of direct-to-consumer advertising $(=1)$ or its acceptance $(=0)$. In turn, $\overline{U A}_{j}, \overline{I N D}_{j}$, $\overline{M A S}_{j}$ and $\overline{L T O}_{j}$ represent respectively the time-invariant uncertainty avoidance, individualism score, masculinity and long-term orientation scores of country $j,{ }^{11}$ while $\overline{G D P}_{j t}$ is the GDP per capita in country $j$ in month $t$. The competition variable $\overline{C O M P}_{i c j t}$ represents the number of competitive molecules that belong to the same ATC category as drug $i$ and that are sold in country $j$ at time $t$. The lagged sales variable $\bar{y}_{i c j t-1}$ represents the sales of the same drug in the same country in $t$ 1. Finally, the introductory lag variable $\overline{I L}_{i c j}$ indicates the number of months elapsed since the first introduction of drug $i$ in any country of the world and its introduction in country $j$. The superscripted bars indicate that variables are standardized (i.e. zero mean, unit variance) across countries and drugs in order to make the effects comparable to each other.

\footnotetext{
${ }^{10}$ The specification of heteroskedastic errors yields a better fit $(L L=-27002 ; \mathrm{BIC}=54304)$ than the specification of homoskedastic errors $(\mathrm{LL}=-27226$; $\mathrm{BIC}=54752)$.

${ }^{11}$ Note that the cultural variables are time-invariant. As noted by Steenkamp et al. (1999), a country's culture is a key environmental characteristic underlying systematic differences in consumer behavior (Triandis 1989). Culture is by nature stable over time and is not supposed to change in the short or medium run.
} 
We also account for heterogeneity across drug categories by including a category-specific intercept $\alpha_{c}$ and we specify the nesting structure among drugs within the same category by including $\gamma_{i c}$. The role of the category- and drug-specific intercepts are to capture a large part of the variation in sales due to the molecules' idiosyncrasies, like e.g. drug indication, marketing instruments used or drug compliance (Bowman et al. 2004). Alternatively, one could have made the time-varying covariate effects $\beta_{1 t}, \ldots, \beta_{13 t}$ drug-specific, but such a specification would exhaust the degrees of freedom and lead to an over-parameterization of the model.

We model the time-varying coefficients $\beta_{1 t}, \ldots, \beta_{13 t}$ in equation (1) using regression splines, which can be seen as a compromise between linear regression and nonparametric regression models. This class of models is also called conditionally parametric models because the time-varying parameters are nonparametric functions whereas the model is parametric for a specified $t$. More specifically, we opt for a penalized splines approach, introduced as P-spline smoothing in Eilers and Marx (1996). Penalized splines build upon recent developments in semiparametric modeling (for an overview of semiparametric models in marketing, see Van Heerde 2000) and has been increasingly advocated and used in statistics journals and books (see e.g. Chiang et al. 2001, Huang et al. 2002, Kauermann 2005, Wu and Zhang 2006).

The advantage of splines compared to the specification of a linear, quadratic or even cubic trend lies in the fact that it does not impose any assumption (linear, quadratic or cubic) as to the interaction of the explanatory variables with time. It constitutes a highly flexible and modular approach by which the time-varying parameters can follow any pattern (Coull et al. 2001, Ruppert et al. 2003), at a low cost in terms of degrees of freedom. Such flexibility is important, given the novelty and the complexity of the research problem, which makes our hypotheses somewhat exploratory. Moreover, some variables may show discontinuities, which suit the semiparametric approach. For instance, the cross-country introduction lag may show discontinuities because of a lack of infrastructure in some lagging countries. We compare our 
results with the estimates from a fully parametric model in the discussion section and find them to lead to very similar findings.

Other estimation methods can be used such as penalized least squares (Hastie and Tibshirani 1993) or local plane fitting (Cleveland et al 1991). Time-varying coefficient models can also be estimated in a bayesian dynamic linear model framework (Neelamegham and Chintagunta 2004, Van Heerde et al. 2004) and/or using Kalman filter (Naik et al. 1998, West et al. 1985, West and Harrison 1997). To account for the dynamic effect of past values of the explanatory variables on sales, as it is the case with dynamic linear models, we include a lagged dependent variable term in equation (1).

The general idea behind splines is that any smoothly varying function can be seen as a linear combination of basis functions. The latter can be polynomial functions of low degree (e.g. linear in case of linear splines, cubic in case of cubic splines). Hence, we can write the timevarying coefficients as

$$
\beta_{p t}=\beta_{p, 0}+\beta_{p, 1} t+\sum_{k=1}^{K} u_{k}^{\beta_{p}}\left(t-\kappa_{k}\right)_{+}, \quad p=1, \ldots, 13
$$

with $\left(t-\kappa_{k}\right)_{+}, k=1, \ldots, K$, a set of $K$ linear spline basis functions, ${ }^{12}$ which are in this case truncated lines with $\kappa_{k}$ the truncation point or knot (i.e. the location where the broken lines are tied together). Such combination of the linear splines basis functions $1, t,\left(t-\kappa_{1}\right)_{+}, \ldots,\left(t-\kappa_{K}\right)_{+}$ gives a piecewise linear function, called spline (see Wedel and Leeflang 1998, for an application of splines in marketing), with $K$ distinct knots at $\kappa_{1}, \ldots, \kappa_{K}$ chosen in the range of $t$, in our case between $t=1$ and $t=84$ months. Alternatively, one could specify quadratic or cubic splines

\footnotetext{
${ }^{12}$ This notation is standard in the statistical literature and indicates that the function equals zero for the values of $t$ where $\left(t-\kappa_{k}\right)_{+}$is negative.
} 
(Sloot et al. 2006) using $1, t, t^{2},\left(t-\kappa_{1}\right)_{+}^{2}, \ldots,\left(t-\kappa_{K}\right)_{+}^{2}$ for quadratic splines, or

$1, t, t^{2}, t^{3},\left(t-\kappa_{1}\right)_{+}^{3}, \ldots,\left(t-\kappa_{K}\right)_{+}^{3}$ for cubic splines.

As we have no prior on the location (on the time axis) where changes in the effect of the explicative variables may occur, we distribute the knots evenly over the complete time period and make sure that we have enough knots to cover the time range. As explained by Sloot et al. (2006), the number of knots should be chosen relative to the number of available observations. While a few knots may result in a model that is too restrictive, too many knots may result in estimation problems. In our application, we use one knot per year that is available, that is $K=7$ knots. ${ }^{13}$

The $u_{k}^{\beta_{p}}$ coefficients can be interpreted as weights to each of the basis functions that constrain their relative influence. These weights are penalized, i.e. they are subject to the constraints $\sum_{k=1}^{K}\left(u_{k}^{\beta_{p}}\right)^{2}<U_{p}$, for some constants $U_{p}, p=1, \ldots, 13$. This penalty induces smoothness in the time-varying effects, preventing fits that are too rough or too wiggly. ${ }^{14}$ Previous research has shown that the model defined by equations (1)-(2), subject to aforementioned constraints, yields fitted values equivalent to those produced by a linear mixed model estimated (e.g. Verbeke and Molenberghs 2000), where the intercepts and slopes $\beta_{p, 0}$ and $\beta_{p, 1}$ in equation (2) are estimated as fixed components and the $u_{k}^{\beta_{p}}$ coefficients are taken as random components with respective variances $\operatorname{Var}\left(u_{k}^{\beta_{p}}\right)=\sigma_{\beta_{p}}^{2}, p=1, \ldots, 13$ (Brumback et al. 1999, Coull et al. 2001, Ruppert et al. 2003). Therefore, the level of smoothing of the timevarying effects is given by $\sigma_{t}^{2} / \sigma_{\beta_{p}}^{2}$. A higher $\sigma_{\beta_{p}}^{2}$ corresponds to a more wiggly function, while

\footnotetext{
${ }^{13}$ Our results turn out to be highly robust to the number and location of knots. In particular, sensitivity checks for $0 \leq K \leq 14$ (see Ruppert et al. 2003) yield very similar findings. When $K=0$, we obtain a linear trend model, which also yields similar fit and conclusions than the model reported in the results section. These results are available from the authors upon request.

${ }^{14}$ This penalization offers the optimal degree of smoothing given the number of knots available. Coefficients are shrunk towards zero. It avoids the resulting spline to interpolate the data points (overfitting or undersmoothing), which would be overly sensitive to measurement errors.
} 
a smaller $\sigma_{\beta_{p}}^{2}$ corresponds to more smoothness. The level of smoothing is determined by residual maximum likelihood (REML) as demonstrated in Wand (2003), rather than using Akaike's information criterion or cross-validation methods. As noted by Opsomer et al. (2001), smoothing parameter selection with any data-driven method typically leads to serious overfitting in case of correlated errors. With linear mixed models however, once the correlation structure is specified, estimation of regression parameters can be carried out without computationally-intensive crossvalidation procedures (Kauermann and Komski 2006, Krivobokova et al. 2006). Linear mixed models are very easy to estimate in standard statistical software (e.g. PROC MIXED in SAS or lme in S-PLUS and R). In addition, the mixed model framework permits the use of likelihood ratio tests for model selection and theory testing. More details can be found in Crainiceanu et al. (2005) or Ruppert et al. (2003).

\section{Instrumenting for Launch Delay}

We deal with the potential endogeneity of the introductory lag variable $\overline{I L}_{i c j}$ in a similar fashion as Desiraju et al. (2004). We find a set of exogenous instruments, denoted by $Z_{i c j}$, that are correlated with the endogenous variable $\overline{I L}_{i c j}$ but uncorrelated with the residuals $\varepsilon_{i c j t}$, and that have an effect on $y_{i c j t}$ only through $\overline{I L}_{i c j}$. As they explained, the underlying idea is to "use variation in $\overline{I L}_{i c j}$ that is explained by the exogenous variables for estimation" as all other unexplained variation is possibly correlated with the residuals. The approach is similar in spirit to two-stage least squares.

In addition to the exogenous variables depicted in equation (1), we consider as instrument for $\overline{I L}_{i c j}$ the introductory lag of all other drugs in the same ATC c (but drug i) in country $j$. This choice is justified by the idea that the introductory lag of the other drugs that belong to the same ATC class $c$ as drug $i$ in a particular country $j$ is a reasonable predictor of the introductory lag of the focal drug in that country. Indeed, we expect that managers' launch decisions would be 
affected by similar underlying factors for all drugs in a given ATC, such as the country's population size or their sales expectations. Moreover, this variable is uncorrelated with the residuals $\varepsilon_{i c j t}$ as confirmed by the high $p$-value obtained from the Durbin-Wu-Haussman test for endogeneity $(p=0.95)$.

Formally, we define the introductory lag of other drugs in the same ATC (but drug i) in country $j$ as $\bar{z}_{-i, c j}$ the mean value of $\overline{I L}_{i c j}$ for all drugs (except the focal drug $i$ ) in that particular country $j$. We construct the instrument as the predicted value from a regression of the endogenous variable $\overline{I L}_{i c j}$ on the entire set of exogenous variables in equation (1) including $\bar{Z}_{-i, c j}$, next denoted as $Z_{i c j}$. Thus, the first stage consists of:

$$
\overline{I L}_{i c j}=\delta_{c}+\tau_{i c}+Z_{i c j} \lambda+\omega_{i c j}
$$

with $\delta_{c}$ and $\tau_{i c}$ the category- and drug-specific intercepts and $\omega_{i c j} \sim N\left(0, \sigma_{\omega}^{2}\right)$. As such, we ensure that $Z_{i c j}$ and $\varepsilon_{i c j t}$ are uncorrelated by construction. The second stage then consists of using in equation (1), the predicted value for $\overline{I L}_{i c j}$ given by estimating equation (3) instead of the endogenous variable $\overline{I L}_{i c j}$. Standard errors are corrected using the results offered by Murphy and Topel (1985).

\section{Results}

This section presents the estimation results of the model described in the previous section. We first discuss results from the instrumental variable procedure described earlier. We then turn to a fit comparison of various specifications (after correcting for endogeneity) and comment on the parameter estimates of the best model.

\section{Instrumental Variable Procedure}

The regression of $\overline{I L}_{i c j}$ on $Z_{i c j}$ in equation (3) provides an approximate adjusted $R^{2}=$ 0.46 and the regression of $\overline{I L}_{i c j}$ on the instrument $\bar{z}_{-i, c j}$ alone gives an approximate adjusted $R^{2}=$ 
0.40 , indicating that our instrument predicts the endogenous variable reasonably well. To further assess whether the instrumental variable procedure works properly, we compare the estimated effect of the introductory lag when the instruments are used or not. We find that, when using instruments instead of the endogenous variable, the estimated effect of the introductory lag variable is on average $29 \%$ higher over the whole time window ( -0.017 instead of -0.025$)$. This result is consistent with our expectations. Recall that managers are expected to make launch decisions to maximize future sales and tend to launch first in countries where they expect higher sales. Thus, an increase in the value of $\varepsilon_{i c j t}$ would lead to an earlier introduction, i.e. a smaller introductory lag, meaning that we should have a negative correlation between $\varepsilon_{i c j t}$ and $\overline{I L}_{i c j}$.

Therefore, if the endogeneity problem remains uncorrected, we would expect the estimated effect for $\overline{I L}_{i c j}$ to be biased downwards, which is consistent with our results.

\section{Model Fit Comparisons}

In Table 3, we evaluate the model fit with respect to alternate specifications, that is: (1) whether to allow parameters to vary over time (none, all or some of them); (2) whether to specify linear, quadratic or cubic spline functions; and (3) whether to include regulatory predictor variables or not. The fourth column of Table 3 reports the number of parameters for the various specifications. ${ }^{15}$

We start our estimation procedure by estimating equation (1)-(2), allowing all effects to vary over time. Our results indicate that this improves model fit. However, when letting all parameters to be time-varying, some of the slope parameters $\beta_{p, 1}$ in equation (2) turn to be insignificant. Therefore, we also estimate a variant of the model in equation (1) with both timevarying and time-invariant effects (i.e. those having a non-significant slope above). The latter gets

\footnotetext{
${ }^{15}$ For instance, the time-invariant model includes 16 parameters: the effect for each of the 13 explanatory variables, as well as $\alpha_{0}, \sigma_{\alpha}^{2}$ and $\sigma_{\gamma}^{2}$. In turn, the time-varying model includes 26 additional parameters as we now add 2 extra parameters for each of the 13 explanatory variables. The derivation of the number of parameters for the other specifications is pretty straightforward.
} 
a better Bayesian Information Criterion (BIC) than a model with time-varying effects only. This result provides evidence that some of the explanatory variables, i.e. physician prescription budgets regulation, direct-to-consumer advertising regulation, uncertainty avoidance, individualism, introductory lag, competition and lagged sales, have a differential impact on the sales of newly launched drugs than on maturing drugs. The other variables appear to have a constant effect over the (observed part of the) product life cycle of new drugs. We present the parameters of this model in Table 4 (time-invariant effects) and Figure 4 (time-varying effects).

Our results also indicate that the specification of quadratic or cubic spline functions does not yield a better fit than linear splines, when accounting for the additional number of parameters such specifications generate.

Finally, the last line of Table 3 provides the fit measures for this model, but excluding the regulatory variables. Such model yields a substantially lower log-likelihood and a much higher $\mathrm{BIC}$, demonstrating that regulatory differences across countries do considerably improve the model fit. In other words, they provide a valuable and substantive explanation as to the crossnational differences in drug sales.

To evaluate the overall fit of the model, we also calculated an approximate adjusted $R^{2}$ measure of the best model, which turns to be is highly satisfactory (adjusted $R^{2}=0.93$ ).

\begin{tabular}{lccccc}
\hline Time-Dependence of the Effects & $\begin{array}{c}\text { Trend } \\
\text { Specification }\end{array}$ & $\begin{array}{c}\text { Regulatory } \\
\text { variables }\end{array}$ & $\begin{array}{c}\text { Number of } \\
\text { Parameters }\end{array}$ & Max LL & BIC \\
\hline Time invariant & - & included & 16 & -27121 & 54402 \\
\hline Time-varying & linear spline & included & 42 & -26982 & 54383 \\
\hline \multirow{2}{*}{ Both time-varying and invariant } & Linear spline & included & $\mathbf{3 0}$ & -27002 & 54304 \\
& quadratic spline & included & 37 & -26992 & 54354 \\
& cubic spline & included & 44 & -26977 & 54392 \\
\cline { 2 - 6 } & linear spline & excluded & 21 & -32317 & 64843 \\
\hline
\end{tabular}

Table 3: A linear splines model with both time-varying and time-invariant effects, including regulatory variables fits the data best. 


\section{Parameter Estimates}

As our model estimates both time-varying and time-invariant parameters, we depict the time-invariant effects in Table 4 and plot the time-varying parameter estimates, with their $95 \%$ confidence bounds in Figure 4 (All results are available in the online appendix).

\begin{tabular}{lcc}
\hline \multicolumn{1}{c}{ Time-Invariant Effects } & Value & Standard Error \\
\hline Manufacturer price regulation & 0.076 & $0.010 * * *$ \\
Marketing efforts to physicians regulation & -0.019 & 0.016 \\
Patient co-payment regulation & -0.050 & 0.037 \\
Masculinity & -0.047 & $0.006 * * *$ \\
Long-term orientation & -0.018 & $0.012 *$ \\
Economic wealth & 0.024 & $0.007 * * *$ \\
\hline BIC $=54304 ; n=21486$ & & \\
${ }^{*} p<0.100 ; * * p<0.050 ; * * * p<0.010$ & &
\end{tabular}

\section{Table 4: Time-invariant coefficients and their respective standard errors.}

We sequentially discuss the effect of (i) regulation, (ii) culture, (iii) economic wealth, (iv) competition, (v) lagged sales and (vi) introductory lag. Our main findings are in line with the theoretical expectations, summarized in Table 1.

Regarding the role of regulation, each of the regulatory variables, except the regulation on marketing efforts to physicians and patient co-payment, is found to have a significant impact on drug sales. In particular, drug sales tend to be higher in countries with manufacturer price controls, than in countries without price controls. While price regulation can cause launch delays (Kyle 2007), it apparently does not constrain drug availability to patients once launched; in fact, we find quite the opposite. However, in contrast to our expectation, we cannot provide strong evidence for any variation over time in the effect of manufacturer price regulation on drug sales. One possible reason why we do not find strong evidence for time variation in this effect may be that prices change rather smoothly over time and thus, may be captured in the diffusion effects (Bass et al. 1994), even if the price in countries with price regulation decreases more than in countries without price regulation. Another possible reason may be the collinearity generated by the inclusion of the interaction between price regulation and time, leading to conservative significance levels. 
Physician prescription budgets

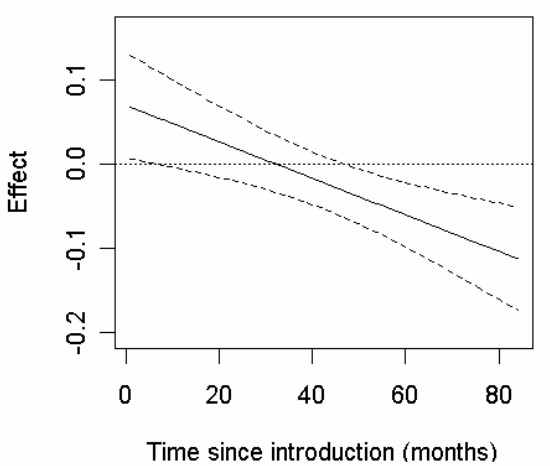

Individualism

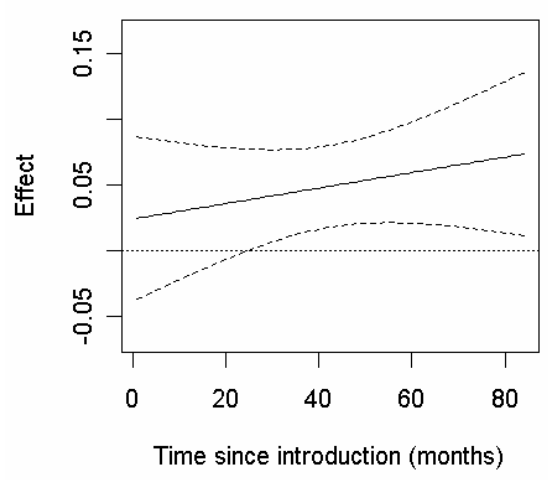

Lagged sales

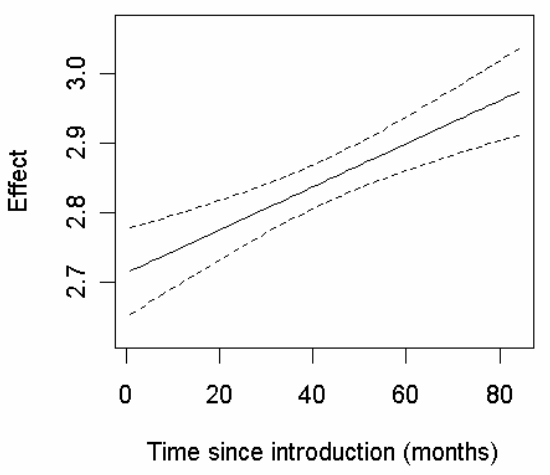

DTCA regulation
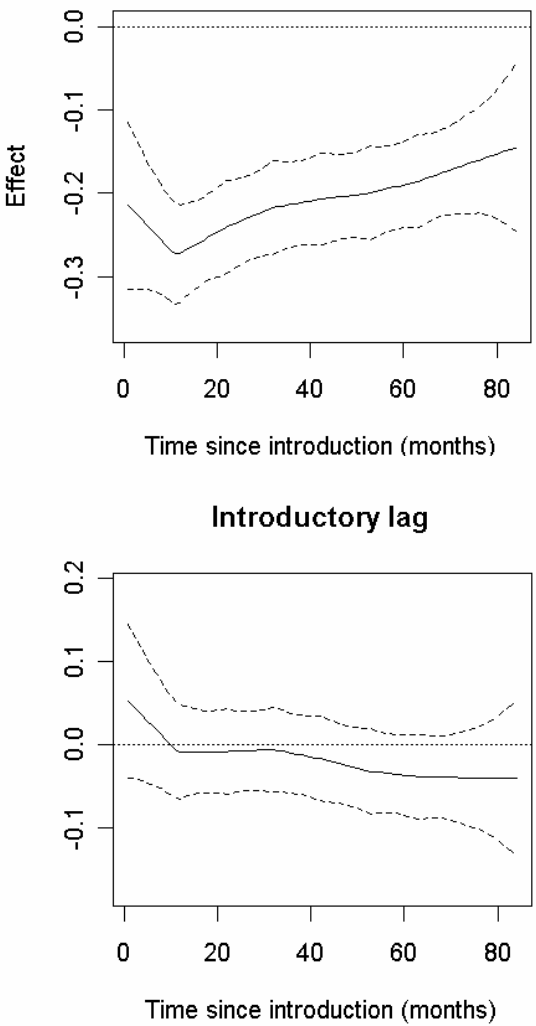

Uncertainty avoidance

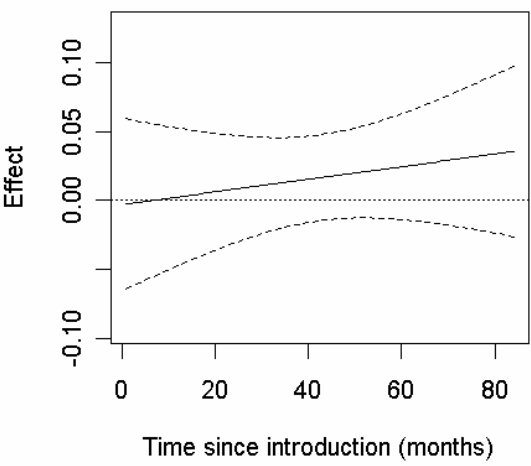

Competition

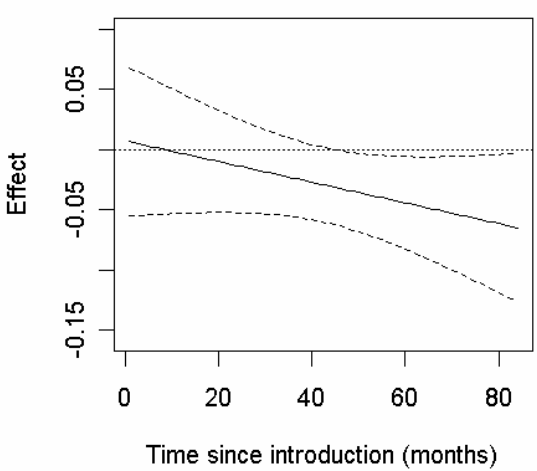

Figure 4: Time-varying effects of the explanatory variables on new drug sales, with $95 \%$ confidence bounds ${ }^{16}$

${ }^{16}$ These confidence bounds are obtained by adding and subtracting twice the estimated standard error of the estimated function (time-varying coefficients; see Ngo and Wand 2004). They can be interpreted as approximate pointwise confidence intervals. In the linear mixed model framework, we derive the standard errors as done in Coull et al. (2001). 
In contrast, the other forms of regulation show a negative effect on drug sales. In particular, regulation on physician prescription budgets has a negative effect on drug sales. However, such regulation appears to be more effective for mature drugs while it has little or no impact on newly launched molecules. This result can be explained by the stepwise prescription behavior of physicians.

While regulating marketing efforts of pharmaceutical firms to physicians tends to have a negative, although non-significant effect, the impact of direct-to-consumer advertising has a highly significant negative effect on new drug sales. This effect appears to be more pronounced for newly launched drugs than for maturing ones. In particular, we find this effect to be the most pronounced around one year after a new drug is introduced.

Co-payment regulation turns out to have a negative and constant effect, although not significant at the $10 \%$ probability level, on drug sales which supports the idea that patients are monetarily penalized for drug usage in co-payment systems. The insignificant effect is likely due to the fact that the presence or absence of such regulation is a rough indicator, compared to the wide variation that exists in co-payment levels.

As to the role of national culture, two of the cultural dimensions, uncertainty avoidance and individualism, tend to have a differential impact on newly launched vs. maturing drugs. In particular, we find that drug sales are higher, although non-significant at the $10 \%$ probability level, in countries with high uncertainty avoidance than in countries with low uncertainty avoidance. This finding can be related to the lower subjective health of inhabitants of uncertainty avoidant nations (Hofstede 2001). It interestingly complements previous research on international diffusion as uncertainty avoidance has been usually found to temper sales growth of new durables. The non-significance of the parameter suggests that the latter effect may actually still play a role, in particular for recently launched drugs for which we find no effect at all. This may result from the relatively higher perceived risk associated with adopting very new drugs. 
However, when time passes, this effect is gradually overwhelmed by the effect of uncertainty avoidance on subjective health.

In addition, we also find that new drug sales are higher in individualist countries than in collectivist countries, which confirms that the former may spend more of their budgets on drugs (as we control for GDP in the estimation) and may seek doctor consultation more actively.

However, we also find that this difference is more pronounced for maturing drugs than for newly launched ones. This result can be explained by the stepwise prescription behavior of physicians.

Furthermore, our results also indicate that new drug sales are lower in masculine countries than in feminine countries, which corroborates our expectations that drug intake by males would be considered a weakness in masculine societies. Given that this argument holds for both new and maturing drugs, we find this effect to be constant over time.

Long-term orientation is found to have a negative, significant, and constant effect on drug sales which supports our hypothesis that patients and physicians in societies high in long-term orientation refrain from classical drug therapy.

Economic wealth, in turn, positively affects new drug sales, which can be related to the fact that wealthier countries have better developed health infrastructure and can afford more easily new drugs than less wealthy countries. As to the effect of competition on new drug sales, we find little negative impact of the number of competitive molecules available in the market. However, this effect turns non-significant for recently introduced molecules. Our results corroborate the findings of Desiraju et al. (2004) for the antidepressant drug category. We do not find evidence that competition among molecules in the same ATC class expands the category. Lagged sales have a significant, positive and increasing effect on sales, which points to the inertia in the physicians' prescription behavior as to the contagion among physicians and patients. Finally, the effect of the introductory lag (when using the instrument) turns to be non-significant at the $5 \%$ probability level, as it is also the case in the global diffusion study of Dekimpe et al. (1998). In line with our expectation, this effect, although non-significant, is the more pronounced 
for recently introduced drugs. Note that the pattern of variation over time of this effect nicely illustrates the potential of P-splines in modeling discontinuous phenomena.

To assess the robustness of our findings, we re-estimate our model with alternate specifications as to the coding of the regulatory variables, the number and the location of knots for the time-varying effects, and the inclusion of interaction effects between the regulatory variables and the ATC categories as well as the lagged sales. Our results turn out to be hardly sensitive to these changes.

\section{Discussion}

This section first discusses the implications our findings have for public policy and managers, after which it turns to the study's limitations and opportunities for future research in this area.

\section{Implications}

An important contribution of this study for both managers and public policy makers is that regulations, in general, matter. Pharmaceutical manufacturer cannot ignore regulatory regimes as they affect the (volume) attractiveness of a country. For instance, drug volumes are ceteris paribus higher in countries with manufacturer price regulation, while they are lower in countries with direct-to-consumer or prescription budget regulation. These insights may be of value to managers when they develop their international launch plans and their expectations towards sales growth in the respective markets they enter. In addition, this study confirms that the cultural and economic characteristics of countries do also affect their attractiveness for pharmaceutical firms. Managers could therefore locate their markets of interest in a map that accounts for regulatory, cultural and economic dimensions that would guide strategy planning.

Our study is also relevant to public policy makers. First, public policy makers are interested in international variation in new drug sales, as they may wish to compare drug consumption - for health care system quality or cost reasons - in their own country to others. 
Second, they wish to know the relationship between regulatory restrictions they enforce and drug consumption. For instance, some regulatory restrictions have a larger effect on newly launched drugs than on maturing drugs, and vice versa. We find sales of maturing drugs to suffer more from prescription budget regulation than newly launched drugs. In contrast, the prohibition of DTCA depresses sales of newly launched drugs more than it depresses sales of maturing drugs. Insights, such as these, provide the regulator with guidelines for the enforcement, monitoring and adaptation of the regulatory environment. Obviously, these latter recommendations need to be treated with caution, in view of the Lucas critique that may apply to our model (Bronnenberg et al. 2005).

\section{Limitations and Future Research}

Similar to most, if not all, studies on international new product growth, we do not study firms' marketing instruments. Our interest in obtaining insights from a very large set of countries that also includes developing countries prohibits the gathering of consistent information on pricing or marketing efforts. Because of the lack of marketing-mix information and the intermediation of marketing-mix on the effects of regulation on sales, our hypotheses are contingent upon marketing-mix effects on sales. The drawback is that our model cannot distinguish between demand (e.g. physician prescription budget regulation may depress drug demand) and supply effects (e.g. restrictions on marketing efforts may restrict the amount of detailing and thereby physician prescriptions). For instance, the mere presence of regulations may "force the hand" of managers and lead to more creative, efficient ways of marketing their products. While our study makes the first steps in exploring the role of regulation in the international sales growth of pharmaceutical drugs, further research that disentangles demand and supply aspects would be of great interest to complement our findings.

In addition, we do not model the effects of the national health infrastructure, such as health expenditures, number of doctors, or hospital beds per capita, neither the effect of the national health status. Yet, health infrastructure and health status are known to be very much 
driven by economic wealth of a country (Desiraju et al. 2004), and we therefore expect it to be partly captured by the effect of GDP per capita in our model.

Third, our dependent variable in this study is sales. We do not discern whether high sales or low sales are good or bad for society, e.g. in terms of over- or underconsumption of drugs. Some have argued that drug usage is too low. For instance, Hassett (2004) illustrates that many deaths can be prevented by higher statin intake, not only in more restrictive countries $(26,000$ preventable deaths in Italy over 5 years $)$, but even in the most intensive drug market $(19,000$ preventable deaths in the United States over 5 years). Others - mostly in Western Europe - have argued that drug usage is typically too high. The study of the influence of country characteristics on patient welfare, rather than new drug sales, may yield interesting insights.

Fourth, our sample of countries is likely biased towards more developed health markets, as the motivation to cover the market for data intermediaries, such as IMS Health, is larger, while the hurdles to do so are smaller. Moreover, independent variables in models such as ours (e.g. the inventory of the regulatory environment) are also more likely to be missing for low income countries than for developing or developed countries. The sample selection issue should lead to some caution towards our results, even though our sample contains more (developing and lowincome) countries than many prior studies in this area.

Fifth, future research on cross-country spill-over effects would also be valuable. Such spill-over effects may occur in: (1) sales - e.g. because physicians affect each other across country borders; (2) marketing instruments - e.g. because manufacturers set their marketing policy on the pan-regional level, rather than the country level; and (3) regulation - e.g. because countries mimic each other in regulatory behavior.

Overall, there is still much research to be done on international growth patterns of new pharmaceutical drugs. Likewise, this study is one of the first endeavors in the role of regulation on international new product growth processes. This area is worthy of more academic attention, given its importance. 


\section{References}

Atun, R., I. Gurol-Urganci. 2006. Impact of regulation on the uptake and diffusion of pharmaceutical innovations: Systematic review. Monograph, Tanaka Business School, Imperial College, London.

Bass, F. M., T. V. Krishnan, D. C. Jain. 1994. Why the Bass model fits without decision variables. Marketing Science 13 (3) 203-223.

Bell, R. A., R. L. Kravitz, M.S. Wilkes. 1999. Direct-to-consumer prescription drug advertising and the public. Journal of General Internal Medicine 14 (11) 651-657.

Berndt, E. R., A. Bhattacharjya, D. N. Mishol, A. Arcelus, T. Lasky. 2002. An analysis of the diffusion of new antidepressants: variety, quality and marketing efforts. Journal of Mental Health Policy and Economics 5 3-19.

Berndt, E. R., P. M. Danzon, G. B. Kruse. 2005. Dynamic competition in pharmaceuticals: Cross-national evidence from new drug diffusion. Working paper. The Wharton School, University of Pennsylvania, Philadelphia, PA.

Bowman, D., C. M. Heilman, P. B. Seetharaman. 2004. Determinants of product-use compliance behavior. Journal of Marketing Research 41 324-338.

Bronnenberg, B. J., P. E. Rossi, N. J. Vilcassim. 2005. Structural modeling and policy simulation. Journal of Marketing Research 42 (February) 22-26.

Brumback, B. A., D. Ruppert, M. P. Wand. 1999. Comment to "Variable selection and function estimation in additive nonparametric regression using a data-based prior." Journal of the American Statistical Association 94 (447) 794-797.

Chandy, R. K., G. J. Tellis, D. J. Macinnis, P. Thaivanich. 2001. What to say when: Advertising appeals in evolving markets. Journal of Marketing Research 38 (November) 399-414.

Chatterjee, R., J. Eliashberg, V. R. Rao. 2000. Dynamic models incorporating competition. In V. Mahajan, E. Muller, Y. Wind (eds.) New Product Diffusion Models 49-73. Kluwer Academic Publishers, Boston, MA.

Chiang, C.-T., J. A. Rice, C. O. Wu. 2001. Smoothing spline estimation for varying coefficient models with repeatedly measured dependent variables. Journal of the American Statistical Association 96 (454) 605619.

Chintagunta, P., R. Desiraju. 2005. Strategic pricing and detailing behavior in international markets. Marketing Science 24 (1) 67-80.

Cleveland, W. S., E. Grosse, W. M. Shyu. 1991. Local regression models. J.M. Chambers and T. Hastie (eds.) Statistical Models in S. Wadworth and Brooks/Cole, Pacific Grove.

Cohen, E.P. 1988. Direct-to-the-public advertisement of prescription drugs. New England Journal of Medicine 318 373-376.

Coull, B. A., D. Ruppert, M. P. Wand. 2001. Simple incorporation of interactions into additive models. Biometrics 57 (2) 539-545.

Crainiceanu, C., D. Ruppert, G. Claeskens, M. P. Wand. 2005. Exact likelihood ratio tests for penalized splines. Biometrika 92 (1) 91-103.

Danzon, P. M., L. Chao. 2000. Cross-national price differences for pharmaceuticals: How large, and why? Journal of Health Economics 19 159-195.

Danzon, P. M., Ketcham J. D. 2003. Reference pricing of pharmaceuticals for Medi-care: Evidence from Germany, the Netherlands and New Zealand. NBER Working Paper. National Bureau of Economic Research, Cambridge, MA.

Danzon, P. M., Y.R. Wang, L. Wang. 2005. The impact of price regulation on the launch delay of new drugs. Health Economics, 14 (3) 269-292.

Dawar, N., P. Parker. 1994. Marketing universals: consumers' use of brand name, price, physical appearance, and retailer reputation as signals of product quality. Journal of Marketing 58 (April) 81-95.

Dekimpe, M. G., D. M. Hanssens. 1999. Sustained spending and persistent response: A new look at long-term marketing profitability. Journal of Marketing Research 36 397-412. 
Dekimpe, M. G., P. M. Parker, M. Sarvary. 1998. Staged estimation of international diffusion models: An application to global cellular telephone adoption. Technological Forecasting and Social Change $57105-$ 132.

Dekimpe, M. G., P. M. Parker, M. Sarvary. 2000. Multimarket and global diffusion. In V. Mahajan, E. Muller, Y. Wind (eds.) New Product Diffusion Models 49-73. Kluwer Academic Publishers, Boston, MA.

Deleersnyder, B., M. G. Dekimpe, J.-B. E. M. Steenkamp, P. S. H. Leeflang. 2008. The role of national culture in advertising's sensitivity to business cycles: An investigation across all continents. ERIM Report Series Reference No. ERS-2007-095-MKT.

Desiraju, R., H. Nair, P. Chintagunta. 2004. Diffusion of new pharmaceutical drugs in developing and developed nations. International Journal of Research in Marketing 21 341-357.

Ding, M., J. Eliashberg. 2008. A dynamic competitive forecasting model incorporating dyadic decision-making, Management Science forthcoming.

Eilers, P. H. C., B. D. Marx. 1996. Flexible smoothing with B-splines and penalties. Statistical Science 11 (2) 89-121.

Ekelund, M., B. Persson. 2003. Pharmaceutical pricing in a regulated market. The Review of Economics and Statistics 85 (2) 298-306.

European Commission. 2004. New products and services: Analysis of regulations shaping new markets. www.cordis.lu/innovation-policy/studies.

Gatignon, H., J. Eliashberg, T. S. Robertson. 1989. Modeling multinational diffusion patterns: An efficient methodology. Marketing Science 8 (3) 231-247.

Gönül, F. F., F. Carter, E. Petrova, K. Srinivasan. 2001. Promotion of prescription drugs and its impact on physicians' choice behavior. Journal of Marketing 65 (July) 79-90.

Grunert, K. G. 1996. Automatic and strategic processes in advertising effects. Journal of Marketing 60 (October) 88-101.

Hahn, M., S. Park, L. Krishnamurthi, A. A. Zoltners. 1994. Analysis of new product diffusion using a foursegment trial-repeat. Marketing Science 13 (3) 224-247.

Hart, J., H. Salman, M. Bergman, V. Neuman, C. Rudniki, D. Gilenberg, A. Matalon, M. Djaldetti. 1997. Do drug costs affect physicians' prescription decisions? Journal of Internal Medicine 241 415-420.

Hassett, K. A. 2004. Price controls and the evolution of pharmaceutical markets. Working paper.

Hastie, T., R. Tibshirani. 1993. Varying-coefficient models. Journal of the Royal Statistical Society, Series B (Methodological) 55 (4) 757-796.

Heeler, R. M., T. P. Hustad. 1980. Problems in predicting new product growth for consumer durables. Management Science 10 (October) 1007-1020.

Helsen, K., K. Jedidi, W. S. DeSarbo. 1993. A new approach to country segmentation utilizing multinational diffusion patterns. Journal of Marketing 57 (October) 60-71.

Hofstede, G. 1980. Culture's consequences: International differences in work-related values. Sage, Beverly Hills, CA.

Hofstede, G. 2001. Culture's consequences: Comparing values, behaviors, institutions, and organizations across nations. $2^{\text {nd }}$ ed. Sage Publications, London, U.K.

Horsky, D., L. S. Simon. 1983. Advertising and the diffusion of new products. Marketing Science 2 (1) 1-17.

Huang, J. Z., C.O. Wu, L. Zhou. 2002. Varying-coefficient models and basis function approximations for the analysis of repeated measurements. Biometrika 89 (1) 111-128.

Jacobzone, S. 2000. Pharmaceutical policies in OECD countries: Reconciling social and industrial goals. OECD Labour Market and Social Policy - Occasional Papers 40.

Janakiraman, R., S. Dutta, C. Sismeiro, P. Stern. 2005. Are they all the same? Physicians' persistence and its implications for marketing communication. Working paper University of Southern California, Los Angeles, U.S.

Kauermann, G. 2005. Penalized spline smoothing in multivariate survival models with varying coefficients. Computational Statistics and Data Analysis 49 (1) 169-186. 
Kauermann, G., P. Komski. 2006. Additive two-way hazards model with varying coefficients. Computational Statistics and Data Analysis 51 (3) 1944-1956.

Kravitz, R. L., R. A. Bell, R. Azari, S. Kelly-Reif, E. Krupat, D. H. Thom. 2003. Direct observation of requests for clinical services in office practice: What do patients want and do they get it? Archives of Internal Medicine 163 1673-1681.

Krishnan, T. V., F. M. Bass, V. Kumar. 2000. Impact of a late entrant on the diffusion of a new product/service. Journal of Marketing Research 37 (2) 269-278.

Krivobokova, T., G. Kauermann, T. Archontakis. 2006. Estimating the term structure of interest rates using penalized splines. Statistical Papers 47 (3) 443-459.

Kumar, V., T.V. Trishnan. 2002. Multinational diffusion models: An alternative framework. Marketing Science 21 (3) 318-330.

Kyle, M. K. 2007. Pharmaceutical price controls and entry strategies. Review of Economics and Statistics 89 (1) 88-99.

Lanjouw, J. O. 2005. Patents, price controls and access to new drugs: How policy affects global market entry. Working paper 61. Center for Global Development, Brookings Institution.

Leeflang, P., J. E. Wieringa, D. R. Wittink. 2005. Modeling the effects of promotion expenditures on the sales of pharmaceuticals. Working paper. University of Groningen.

Lilien, G. L., J. D. C. Little. 1976. The ADVISOR project: A study of industrial marketing budgets. Sloan Management Review 17 (Spring) 17-31.

Lynn, M., G. M. Zinkhan, J. Harris. 1993. Consumer tipping: a cross-country study. Journal of Consumer Research 20 (December) 478-488.

Mahajan, V., E. Muller. 1994. Innovation diffusion in a borderless global market: Will the 1992 unification of the European community accelerate diffusion of new ideas, products and technologies? Technological Forecasting and Social Change 45 221-235.

Mahajan, V., S. Sharma, R. D. Buzzell. 1993. Assessing the Impact of Competitive Entry on Market Expansion and Incumbent Sales. Journal of Marketing 57 (3) 39-52.

Manchanda, P., P. K. Chintagunta. 2004. Responsiveness of physician prescription behavior to salesforce effort: an individual level analysis. Marketing Letters 15 (2-3) 129-145.

Manchanda, P., E. Honka. 2005. The effects and role of direct-to-physician marketing in the pharmaceutical industry: an integrative review. Yale Journal of Health Policy, Law and Economics 5 785-822.

Manchanda, P., P. E. Rossi, P. K. Chintagunta. 2004. Response modeling with non-random marketing mix variables. Journal of Marketing Research 41 November 467-478.

Mantrala, M. K., P. Sinha, A. A. Zoltners. 1994. Structuring a multiproduct sales quota bonus plan for a heterogeneous sales force. Marketing Science 13 (2) 121-144.

Mason, C. H. 1990. New product entries and product class demand. Marketing Science 9 (1) 58-73.

Mehta, A., S.C. Purvis. 2003. Consumer response to print prescription drug advertising. Journal of Advertising Research 43 (2) 194-206.

Michaut, A. 2004. Consumer response to innovative products: with application to foods. $P h D$ thesis. Wageningen University.

Mintzes, B., M. L. Barer, R. L. Kravitz, B. Bassett, J. Lexchin, A. Kazanjian, R. G. Evans, R. Pan, S. A. Marion. 2003. How does direct-to-consumer advertising (DTCA) affect prescribing? A survey in primary care environments with and without legal DTCA. Canadian Medical Association Journal 169 (5) 405-412.

Mizik, N., R. Jacobson. 2004. Are physicians 'easy marks'? Quantifying the effects of detailing and sampling on new prescriptions. Management Science 50 (12) 1704-1715.

Murphy, K. M., R. H. Topel. 1985. Estimation and inference in two-step econometric models. Journal of Business \& Economic Statistics 3 (4) 370-379.

Naik, P. A, M. K Mantrala, A. G. Sawyer. 1998. Planning media schedules in the presence of dynamic advertising quality. Marketing Science 17 (3) 214-35.

Narayanan, S. 2004. Heterogeneous learning and the targeting of marketing communication for new products. Working paper. 
Narayanan, S., R. Desiraju, P. K. Chintagunta. 2004. Return on investment implications for pharmaceutical promotional expenditures: the role of marketing-mix interactions. Journal of Marketing 68 (October) 90105.

Narayanan, S., P. Manchanda, P. K. Chintagunta. 2005. Temporal differences in the role of marketing communication in new product categories. Journal of Marketing Research 42 278-290.

Narayanan, S., P. Manchanda. 2006. The role of free samples in the pharmaceutical industry: An empirical analysis. 2006 Informs Marketing Science Conference. University of Pittsburgh.

Neelamegham, R., P. K. Chintagunta. 2004. Modeling and forecasting the sales of technology products. Quantitative Marketing and Economics 2 195-232.

Neslin, Scott. 2001. ROI analysis of pharmaceutical promotion. Unpublished study conducted for the Association of Medical Publications available at http://www.rappstudy.org.

Ngo, L., M.P. Wand. 2004. Smoothing with mixed model software. Journal of Statistical Software 9 (1) 1-54.

Opsomer, J., Y. Wang, Y. Yang. 2001. Nonparametric regression with correlated errors. Statistical Science 16 134-153.

Parsons, L. J., P. Vanden Abeele. 1981. Analysis of sales call effectiveness. Journal of Marketing Research 18 (February) 107-113.

Prosser, H., T. Walley. 2003. New drug uptake: Qualitative comparison of high and low prescribing GP's attitudes and approach. Family Practice 20 (5) 583-591.

Prosser, H., S. Almond, T. Walley. 2003. Influences on GP's decision to prescribe new drugs - the importance of who says what. Family Practice 20 (1) 61-68.

Reuveni, H., B. Sheizaf, A. Elhayany, M. Sherf, Y. Limoni, S. Scharff, R. Peled. 2002. The effect of drug copayment policy on the purchase of prescription drugs for children with infections in the community. Health Policy 62 (1) 1-13.

Rosenthal, M. B., E. R. Berndt, J. M. Donohue, R. G. Frank, A. M. Epstein. 2002. Promotion of prescription drugs to consumers. The New England Journal of Medicine 346 (7) 498-505.

Rosenthal, M. B., E. R. Berndt, J. M. Donohue, A. M. Epstein, R. G. Frank. 2003. Demand effects of recent changes in prescription drug information. D. M. Cutler, A. M. Garber (eds.) Frontiers in Health Policy Research 6 1-26. MIT Press, Cambridge, MA.

Roth, M. 1995. The effects of culture and socioeconomics on the performance of global brand image strategies. Journal of Marketing Research 32 (May) 163-175.

Ruppert, D., M. P. Wand, R. J. Carroll. 2003. Semiparametric Regression. Cambridge Series in Statistical and Probabilistic Mathematics, Cambridge University Press, Cambridge, U.K.

Schafer, J. L. 1997. Analysis of Incomplete Multivariate Data (Chapter 5). Chapman and Hall, CRC Press. London, U.K.

Schafer, J. L., J. W. Graham. 2002. Missing data: Our view of the state of the art. Psychological Methods 7 (2) 147-177.

Schwartz, R. K., S. B. Soumerai, J. Avorn. 1989. Physician motivations for nonscientific drug prescribing. Social Science and Medicine 28 (6) 577-582.

Schwartz, S. H. 1992. Universals in the content and structure of values: theoretical advances and empirical tests in 20 countries. M. Zanna (ed.) Advances in Experimental Social Psychology 25 1-65. Academic Press, Orlando, FL.

Shugan, S. M. 2003. Editorial: Defining interesting research problems. Marketing Science 22 (1) 1-15.

Sloot, L. M., D. Fok, P. C. Verhoef. 2006. The short- and long-term impact of an assortment reduction on category sales. Journal of Marketing Research 43 536-548.

Steenkamp, J.-B. E.M., F. ter Hofstede, M. Wedel. 1999. A cross-national investigation into the individual and national cultural antecedents of consumer innovativeness. Journal of Marketing 63 (April) 55-69.

Stremersch, S., G. J. Tellis. 2004. Understanding and managing international growth of new products. International Journal of Research in Marketing 21 421-438.

Takada, H., D. Jain. 1991. Cross-national analysis of diffusion of consumer durable goods in Pacific Rim countries. Journal of Marketing 55 (April) 48-54. 
Talukdar, D., K. Sudhir, A. Ainslie. 2002. Investigating new product diffusion across products and countries. Marketing Science 21 (1) 97-114.

Tellis, G. J., C. Fornell. 1988. The relationship between advertising and product quality over the product life cycle: A contingency theory. Journal of Marketing Research 25 (February) 64-71.

Tellis, G. J., S. Stremersch, E. Yin. 2003. The international takeoff of new products: The role of economics, culture, and country innovativeness. Marketing Science 22 (2) 188-208.

The Economist. 2007. From bench to bedside - An unhealthy burden. June 30.

Triandis, H. C. 1989. The self and social behavior in differing cultural contexts. Psychological Review 96 (July) 506-520.

Urch. 2005. The Guide to Pharmaceutical Pricing and Reimbursement Systems: Western Europe. Urch Publishing Ltd, London, U.K.

Vakratsas, D., C. Kolsarici. 2007. The effects of generic and branded advertising messages for a new prescription pharmaceutical: Assessment of a "regulation experiment". Working paper.

Van den Bulte, C., S. Stremersch. 2004. Social contagion and income heterogeneity in new product diffusion: A meta-analytic test. Marketing Science 23 (4) 530-544.

Van Heerde, H. J. 2000. Non- and semiparametric regression models. P. S. H. Leeflang, D. R. Wittink, M. Wedel, P. A. Naert. Building Models for Marketing Decisions 396-408. Kluwer Academic Publishers, Boston, MA.

Van Heerde, H. J., C. F. Mela, P. Manchanda. 2004. The dynamic effect of innovation on market structure. Journal of Marketing Research 41 (2) 166-183.

Venkataraman, S., S. Stremersch. 2007. The debate on influencing doctors' decisions: Are drug characteristics the missing link? Management Science 53 (11) 1688-1701.

Verbeke, G., G. Molenberghs. 2000. Linear Mixed Models for Longitudinal Data. Springer-Verlag, New York, NY.

Wand, M. P. 2003. Smoothing and mixed models. Computational Statistics 18 223-249.

Weber, B. A., B. L. Roberts, G. J. McDougall, Jr. 2000. Exploring the efficacy of support groups for men with prostate cancer. Geriatric Nursing 21 (5) 250-253.

Wedel, M., P. S. H. Leeflang. 1998. A model for the effects of psychological pricing in Gabor-Granger price studies. Journal of Economic Psychology 19 (2) 237-260.

Weissman, J. S., D. Blumenthal, A. J. Silk, K. Zapert, M. Newman, R. Leitman. 2004. Consumers' reports on the health effects of direct-to-consumer drug advertising. Health Affairs - Web Exclusive W3 82-95.

West, D. 1999. Mixing it up in the media. Pharmaceutical Executive DTC Times Supplement 2 (9) 6.

West, M., P. J. Harrison, H. S. Migon. 1985. Dynamic generalized linear models and Bayesian forecasting. Journal of the American Statistical Association 80 73-97.

West, M., J. P. Harrison. 1997. Bayesian Forecasting and Dynamic Models. Springer-Verlag, New York, NY.

Wosinska, M. 2002. Just what the patient ordered? Direct-to-consumer advertising and the demand for pharmaceutical products. HBS Marketing Research Paper Series 02-04.

Wu, H., J.-T. Zhang. 2006. Nonparametric Regression Methods for Longitudinal Data: Mixed-Effects Modeling Approaches. Wiley, New York, NY.

Wright, D. J. 2004. The drug bargaining game: Pharmaceutical regulation in Australia. Journal of Health Economics 23 785-813. 


\section{Online Appendix}

This technical appendix provides additional information about: (1) the computation of the typical patterns of pricing and marketing efforts in Figures 1 and 2 and (2) additional results on our model estimates.

\section{Computation of the Typical Patterns of Pricing and Marketing Efforts}

Let $R_{j}$ be a dummy variable indicating whether a regulation is applied in a country $j$, with $R_{j}=1$ if a price regulation is enforced in country $j$, and $R_{j}=0$ otherwise. Following a similar framework as Coull et al. (2001), we model price $_{\text {icjt }}$ as follows:

$$
\text { price }_{i c j t}=f_{R_{j}}(t)+\varepsilon_{i c j t}, \quad \varepsilon_{i c j t} \sim N\left(0, \sigma_{t}^{2}\right) .
$$

where $f_{R_{j}=0}(t)$ and $f_{R_{j}=1}(t)$ respectively represent the typical pricing pattern in a country where a manufacturer price regulation is applied, respectively the typical pricing pattern in a country where no price regulation is applied. A linear penalized spline model (see Model Specification section for more details) can be written as

$$
\text { price }_{i c j t}=\beta_{0}+\beta_{1} t+\sum_{k=1}^{K} v_{k}\left(t-\kappa_{k}\right)_{+}+R_{j}\left\{\left(\gamma_{0}+\gamma_{1} t\right)+\sum_{k=1}^{K} w_{k}\left(t-\kappa_{k}\right)_{+}\right\}+\varepsilon_{i c j t}
$$

with random coefficients $v_{k} \sim N\left(0, \sigma_{v}^{2}\right)$ and $w_{k} \sim N\left(0, \sigma_{w}^{2}\right)$. We use a similar framework to model the typical pattern of marketing efforts in a country where a regulation that restrict marketing efforts to physicians is applied and in a country where it is not the case. 


\section{Model Estimates}

The following Table A.1. presents all estimates, in full, of the best model, of which we presented the core results in Table 4 and Figure 4.

\section{a) Fixed Effects}

\begin{tabular}{lcccc} 
& \multicolumn{2}{c}{ Intercepts $\beta_{p, 0}$} & \multicolumn{2}{c}{ Slopes $\beta_{p, 1}$} \\
\cline { 2 - 5 } & Estimate & $\begin{array}{c}\text { Standard } \\
\text { Error }\end{array}$ & Estimate & $\begin{array}{c}\text { Standard } \\
\text { Error }\end{array}$ \\
\cline { 2 - 5 } Regulatory variables & & & & \\
Manufacturer price regulation & & & & - \\
Marketing efforts to physicians regulation & 0.076 & 0.010 & - & - \\
Regulation on the physician prescription budgets & -0.019 & 0.016 & - & 0.001 \\
Patient co-payment regulation & 0.070 & 0.017 & -0.002 & - \\
DTCA regulation & -0.050 & 0.037 & - & 0.008 \\
Cultural dimensions & -0.209 & 0.092 & -0.006 & \\
$\quad$ Uncertainty avoidance & & & & 0.000 \\
Individualism & -0.003 & 0.008 & 0.000 & - \\
Masculinity & 0.024 & 0.016 & 0.001 & 0.000 \\
Long-term orientation & -0.047 & 0.006 & - & - \\
Economic wealth & -0.018 & 0.012 & - & - \\
Competition & 0.024 & 0.007 & - & 0.000 \\
Lagged sales & 0.007 & 0.008 & -0.001 & 0.001 \\
Introductory lag (IV) & 2.713 & 0.026 & 0.003 & 0.001 \\
\hline
\end{tabular}

\section{b) Random Effects}

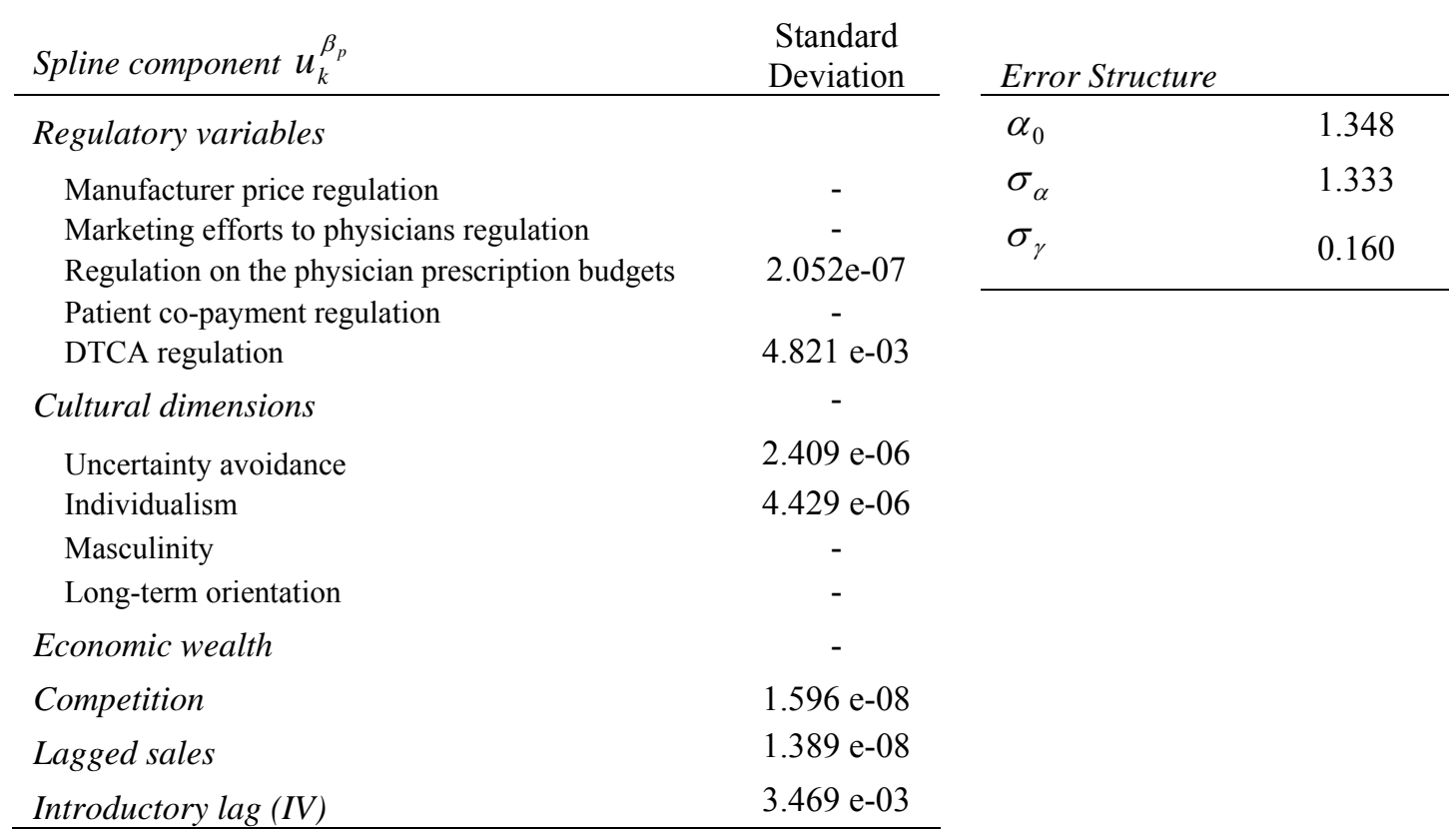

Table A.1: Estimation results of the best time-varying coefficient model 


\section{Publications in the Report Series Research ${ }^{*}$ in Management}

\section{ERIM Research Program: "Marketing"}

2008

Experts' Stated Behavior

Youssef Boulaksil and Philip Hans Franses

ERS-2008-001-MKT

http://hdl.handle.net/1765/10900

The Value of Analogical Reasoning for the Design of Creative Sales Promotion Campaigns: A Case-Based Reasoning Approach

Niek A.P. Althuizen and Berend Wierenga

ERS-2008-006-MKT

http://hdl.handle.net/1765/11289

Shopping Context and Consumers' Mental Representation of Complex Shopping Trip Decision Problems Benedict G.C. Dellaert, Theo A. Arentze and Harry J.P. Timmermans ERS-2008-016-MKT

http://hdl.handle.net/1765/11812

Modeling the Effectiveness of Hourly Direct-Response Radio Commercials

Meltem Kiygi Calli, Marcel Weverbergh and Philip Hans Franses

ERS-2008-019-MKT

http://hdl.handle.net/1765/12242

Choosing Attribute Weights for Item Dissimilarity using Clikstream Data with an Application to a Product Catalog Map Martijn Kagie, Michiel van Wezel and Patrick J.F. Groenen

ERS-2008-024-MKT

http://hdl.handle.net/1765/12243

The Effect of Superstar Software on Hardware Sales in System Markets

Jeroen L.G. Binken and Stefan Stremersch

ERS-2008-025-MKT

http://hdl.handle.net/1765/12339

Sales Growth of New Pharmaceuticals Across the Globe: The Role of Regulatory Regimes

Stefan Stremersch and Aurélie Lemmens

ERS-2008-026-MKT

http://hdl.handle.net/1765/12340

\footnotetext{
A complete overview of the ERIM Report Series Research in Management: https://ep.eur.nl/handle/1765/1

ERIM Research Programs:

LIS Business Processes, Logistics and Information Systems

ORG Organizing for Performance

MKT Marketing

F\&A Finance and Accounting

STR Strategy and Entrepreneurship
} 